\title{
ORDINARY AND ALMOST ORDINARY PRYM VARIETIES
}

\author{
EKIN OZMAN AND RACHEL PRIES
}

\begin{abstract}
We study the $p$-rank stratification of the moduli space of Prym varieties in characteristic $p>0$. For arbitrary primes $p$ and $\ell$ with $\ell \neq p$ and integers $g \geq 3$ and $0 \leq f \leq g$, the first theorem generalizes a result of Nakajima by proving that the Prym varieties of all the unramified $\mathbb{Z} / \ell$-covers of a generic curve $X$ of genus $g$ and $p$-rank $f$ are ordinary. Furthermore, when $p \geq 5$ and $\ell=2$, the second theorem implies that there exists a curve of genus $g$ and $p$-rank $f$ having an unramified double cover whose Prym has $p$-rank $f^{\prime}$ for each $\frac{g}{2}-1 \leq f^{\prime} \leq g-2$; (these Pryms are not ordinary). Using work of Raynaud, we use these two theorems to prove results about the (non)-intersection of the $\ell$-torsion group scheme with the theta divisor of the Jacobian of a generic curve $X$ of genus $g$ and $p$-rank $f$.

Keywords: Prym, curve, abelian variety, Jacobian, $p$-rank, theta divisor, torsion point, moduli space.
\end{abstract}

MSC: primary: $11 \mathrm{G} 10,14 \mathrm{H} 10,14 \mathrm{H} 30,14 \mathrm{H} 40,14 \mathrm{~K} 25$

secondary: 11G20, 11M38, 14H42, 14K10, 14K15.

\section{INTRODUCTION}

Suppose $X$ is a smooth projective connected curve of genus $g \geq 2$ defined over an algebraically closed field $k$ of characteristic $p>0$. Suppose $\pi: Y \rightarrow X$ is an unramified cyclic cover of degree $\ell$ for some prime $\ell \neq p$. Then $Y$ has genus $g_{Y}=\ell(g-1)+1$ by the Riemann-Hurwitz formula. For each of the $\ell^{2 g}-1$ unramified $\mathbb{Z} / \ell$-covers $\pi: Y \rightarrow X$, the Jacobian $J_{Y}$ is isogenous to $J_{X} \oplus P_{\pi}$ for an abelian variety $P_{\pi}$ of dimension $(\ell-1)(g-1)$, called the Prym variety of $\pi$. In particular, when $\ell=2$ and $\pi: Y \rightarrow X$ is an unramified double cover, then $Y$ has genus $2 g-1$ and $P_{\pi}$ is a principally polarized abelian variety of dimension $g-1$.

In this paper, we study the relationship between the $p$-ranks of $J_{X}$ and $P_{\pi}$. The $p$-rank $f_{A}$ of an abelian variety $A / k$ of dimension $g_{A}$ is the integer $0 \leq f_{A} \leq g_{A}$ such that the number of $p$-torsion points in $A(k)$ is $p^{f_{A}}$. One says that $A$ is ordinary if its $p$-rank is as large as possible $\left(f_{A}=g_{A}\right)$ and is almost ordinary if its $p$-rank equals $g_{A}-1$.

Consider the moduli space $\mathcal{M}_{g}$ whose points represent smooth curves $X$ of genus $g$ and the moduli space $\mathcal{R}_{g, \ell}$ whose points represent unramified $\mathbb{Z} / \ell$-covers $\pi: Y \rightarrow X$. There is a finite flat morphism of degree $\ell^{2 g}-1$, denoted

$$
\Pi_{\ell}: \mathcal{R}_{g, \ell} \rightarrow \mathcal{M}_{g}
$$

which takes the point representing a cover $\pi: Y \rightarrow X$ to the point representing the curve $X$ [8, Page 6]. For $0 \leq f \leq g$, let $\mathcal{M}_{g}^{f}$ denote the $p$-rank $f$ stratum of $\mathcal{M}_{g}$. For most $g$ and $f$, it is not known whether $\mathcal{M}_{g}^{f}$ is irreducible; however, every component of $\mathcal{M}_{g}^{f}$ has dimension $2 g-3+f[9$, Theorem 2.3].

The first author was partially supported by AWM-NSF Mentoring Travel Grant 2013, TUBITAK 2232 fellowship 114C126 and Bogazici University Research Grant 15B06SUP3. The second author was partially supported by grants NSF DMS-15-02227 and NSA 131011. We would like to thank Achter, Bruin, CasalainaMartin, Farkas, and Grushevsky for good conversations, and the referees for insightful comments. 
By a result of Nakajima, the Prym varieties of the unramified $\mathbb{Z} / \ell$-covers of the generic curve $X / k$ of genus $g \geq 2$ are ordinary [15, Theorem 2]. In other words, the cover represented by the generic point of $\mathcal{R}_{g, \ell}$ has an ordinary Prym.

The first theorem in the paper generalizes Nakajima's result by adding a condition on the $p$-rank of $X$. Specifically, if $X$ is a generic $k$-curve of genus $g$ and $p$-rank $f$, then Theorem 1.1 implies that the Prym varieties of all of the unramified $\mathbb{Z} / \ell$-covers of $X$ are ordinary.

Raynaud used the theta divisor $\Theta_{X}$ in $J_{X}$ to study the $p$-rank of Prym varieties [17, 18, 19]. (See Section 8 for the definition of $\Theta_{X}$.) Using Raynaud's work, our theorems yield new results about the (non)-existence of points of order $\ell$ contained in $\Theta_{X}$.

Theorem 1.1. Let $\ell \neq p$ be prime. Let $g \geq 2$ and $0 \leq f \leq g$ with $f \neq 0$ if $g=2$. Let $S$ be an irreducible component of $\mathcal{M}_{g}^{f}$.

(1) (See Theorem 4.5) Then $\Pi_{\ell}^{-1}(S)$ is irreducible (of dimension $2 g-3+f$ ) and the cover represented by the generic point of $\Pi_{\ell}^{-1}(S)$ has an ordinary Prym.

(2) (See Theorem 8.3) If $X$ is the curve represented by the generic point of $S$, then the theta divisor $\Theta_{X}$ of the Jacobian of $X$ does not contain any point of order $\ell$.

The second theorem demonstrates the existence of unramified double covers $\pi: Y \rightarrow X$ such that the Prym $P_{\pi}$ is almost ordinary (with $p$-rank $f^{\prime}=g-2$ ).

Theorem 1.2. Let $\ell=2$. Let $g \geq 2$ and $0 \leq f \leq g$ (with $f \geq 2$ when $p=3$ ). Let $S$ be an irreducible component of $\mathcal{M}_{g}^{f}$.

(1) (See Theorem 7.1) The locus of points of $\Pi_{2}^{-1}(S)$ representing covers whose Prym $P_{\pi}$ is almost ordinary is non-empty with codimension 1 in $\Pi_{2}^{-1}(S)$.

(2) (See Theorem 8.4) The locus of points of $S$ representing curves $X$ for which $\Theta_{X}$ contains a point of order 2 is non-empty with codimension 1 in $S$.

As an application of Theorem 1.2 , we prove:

Application 1.3. (See Corollary 7.3) Let $\ell=2$ and $p \geq 5$. Let $g \geq 2$ and $0 \leq f \leq g$. Then there exists a smooth curve $X / \overline{\mathbb{F}}_{p}$ of genus $g$ and $p$-rank $f$ having an unramified double cover $\pi: Y \rightarrow X$ for which the Prym has $p$-rank $f^{\prime}$ for each $\frac{g}{2}-1 \leq f^{\prime} \leq g-1$.

Here is an outline of the paper. Section 2 contains background about Prym varieties and the $p$-rank stratification of $\mathcal{M}_{g}$. In Section 3, we analyze the $p$-ranks of Pryms of covers of singular curves and the $p$-rank stratification of the boundary of $\mathcal{R}_{g, \ell}$.

Section 4 contains the proof of Theorem 1.1(1). The proof mirrors Nakajima's technique of degeneration to the boundary of $\mathcal{R}_{g, \ell}$; the argument is more complicated, however, because $\mathcal{M}_{g}^{f}$ may not be irreducible. To avoid this difficulty, for each irreducible component $S$ of $\mathcal{M}_{g}^{f}$, we consider the $\mathbb{Z} / \ell$-monodromy of the tautological curve $X \rightarrow S$, namely the image of the fundamental group $\pi_{1}\left(S, s_{0}\right)$ in $\operatorname{Aut}\left(\operatorname{Pic}^{0}(X)[\ell]_{s_{0}}\right)$. A key point is that the $\mathbb{Z} / \ell$-monodromy of $X \rightarrow S$ is as large as possible, namely $\mathrm{Sp}_{2 g}(\mathbb{Z} / \ell)[1$, Theorem 4.5]. We use this to prove that $\Pi_{\ell}^{-1}(S)$ is irreducible and that it degenerates to a particular boundary component $\Delta_{i: g-i}$.

In Sections 5 77, we restrict to the case $\ell=2$. In Section 5, we stratify $\mathcal{R}_{g, 2}$ by $\left(f, f^{\prime}\right)$ where $f$ (resp. $f^{\prime}$ ) is the $p$-rank of $X$ (resp. $P_{\pi}$ ). Using purity, we prove that the dimension of each component of the $\left(f, f^{\prime}\right)$ stratum of $\mathcal{R}_{g, 2}$ is at least $g-2+f+f^{\prime}$ Proposition 5.2. Section 6 contains results about non-ordinary Pryms in the low genus cases $g=2,3$, generalizing $[9$ Theorem 6.1]. 
Section 7 contains the proof of Theorem 1.2(1). The proof is again an inductive argument which uses the boundary component $\Delta_{i: g-i}$, but it relies on more refined information from each of Sections 3, 4, 5, and 6.

Application 1.3 (Corollary 7.3) follows from Theorems 1.1 and 1.2 using a 'straightforward' deformation argument for $f^{\prime} \leq g-3$ : Suppose $\pi_{s}$ is an unramified double cover of a singular curve of genus $g$ and $p$-rank $f$ for which the Prym has $p$-rank $f^{\prime}$. Applying [1, Section 3], one can deform $\pi_{s}$ to an unramified double cover of a smooth curve of genus $g$ whose $p$-rank is still $f$. However, it is possible that the $p$-rank $f^{\prime}$ of the Prym increases in this deformation. In fact, there are situations where this is guaranteed to happen, see Remark 6.3. Under the hypotheses of Corollary 7.3, we construct a deformation of $\pi_{s}$ for which the $p$-rank of the Prym remains constant. We emphasize that the technique used in Theorems 1.1-1.2 is stronger than the straight-forward approach and gives more information about the $p$-rank stratification of $\mathcal{R}_{g, \ell}$.

Section 8 contains the definition of the theta divisor $\Theta_{X}$ and the proofs of Theorems $1.1(2)$ and 1.2 (2). We then compare our results with those of Raynaud [17, 18] and Pop/Saidi [16]. Briefly, Raynaud's results are stronger in that they apply to an arbitrary base curve $X$ but are weaker in other ways: his result about ordinary Pryms applies only when $\ell>(p-1) 3^{g-1} g$ !, in which case he shows that at least one of the Pryms is ordinary; and, in his result for non-ordinary Pryms, the Galois group of the cover is solvable but not cyclic and the $p$-rank of the Prym is not determined. In [16, Proposition 2.3], the result is stronger in that it applies to an arbitrary curve which is either non-ordinary or whose Jacobian is absolutely simple, but is weaker in that the degree of the cyclic unramified cover and the $p$-rank of the Prym are not determined.

Section 9 contains some open questions. For example, in Section 9.1, we illustrate the difficulty in proving these results computationally, even for $g=2$ and a fixed small prime $p$. See [4] for results about $p$-ranks of ramified cyclic covers of curves.

\section{PRYM VARIETIES AND $p$-RANK STRATIFICATIONS}

Suppose $X$ is a smooth projective curve of genus $g$ defined over $k$. The Jacobian $J_{X}$ of $X$ is a principally polarized abelian variety of dimension $g$. A $\mathbb{Z} / \ell$-cover $\pi: Y \rightarrow X$ is a Galois cover together with an isomorphism $\iota: \operatorname{Gal}(Y / X) \rightarrow \mathbb{Z} / \ell$. For a prime $\ell \neq p$, there is a bijection between points of order $\ell$ on $J_{X}$ and unramified connected $\mathbb{Z} / \ell$-covers $\pi: Y \rightarrow X$.

2.1. Prym varieties. Suppose $\pi: Y \rightarrow X$ is an unramified $\mathbb{Z} / \ell$-cover. The Prym variety $P_{\pi}$ is the connected component containing 0 of the norm map on Jacobians. More precisely, if $\sigma$ is the endomorphism of $J_{Y}$ induced by a generator $\sigma$ of $\operatorname{Gal}(Y / X)$, then

$$
P_{\pi}=\operatorname{Im}(1-\sigma)=\operatorname{ker}\left(1+\sigma+\ldots+\sigma^{\ell-1}\right)^{0} .
$$

The canonical principal polarization of $J_{Y}$ induces a polarization on $P_{\pi}$ [14, Page 6]. This polarization is principal when $\ell=2$.

2.2. Moduli spaces of unramified cyclic covers. Let $\mathcal{R}_{g, \ell}$ denote the moduli space whose points represent unramified $\mathbb{Z} / \ell$-covers of smooth projective curves of genus $g$; it is a smooth Deligne-Mumford stack [7, Page 5].

The points of $\mathcal{R}_{g, \ell}$ can also represent triples $(X, \eta, \phi)$ where $X$ is a smooth genus $g$ curve equipped with a line bundle $\eta \in \operatorname{Pic}(X)$ and an isomorphism $\phi: \eta^{\otimes \ell} \stackrel{\sim}{\rightarrow} \mathcal{O}_{X}$. This is because the data of the $\mathbb{Z} / \ell$-cover $\pi: Y \rightarrow X$ is equivalent to the data $(X, \eta, \phi)$. 
The morphism $\Pi_{\ell}: \mathcal{R}_{g, \ell} \rightarrow \mathcal{M}_{g}$, which sends the point representing $\pi: Y \rightarrow X$ to the point representing $X$, is surjective, étale, and finite of degree $\ell^{2 g}-1$. Thus $\operatorname{dim}\left(\mathcal{R}_{g, \ell}\right)=3 g-3$.

2.3. Marked covers. A point of $\mathcal{M}_{g ; 1}$ represents a smooth curve $X$ of genus $g$ together with a marking, namely the choice of a point $x \in X$. A point of $\mathcal{R}_{g, \ell ; 1}$ represents an unramified $\mathbb{Z} / \ell$-cover $\pi: Y \rightarrow X$, where $X$ is a smooth curve of genus $g$, together with a marking $x^{\prime} \mapsto x$, namely the choice of a point $x^{\prime} \in \pi^{-1}(x)$. The marking $x^{\prime} \mapsto x$ determines a labeling of the $\ell$ points of the fiber $\pi^{-1}(x)$ because of the $\mathbb{Z} / \ell$-action. There are forgetful maps $\psi_{M}: \mathcal{M}_{g ; 1} \rightarrow \mathcal{M}_{g}$ and $\psi_{R}: \mathcal{R}_{g, \ell ; 1} \rightarrow \mathcal{R}_{g, \ell}$.

Lemma 2.1. If $S \subset \mathcal{M}_{g}$ is irreducible, then $\psi_{M}^{-1}(S)$ is irreducible in $\mathcal{M}_{g ; 1}$. If $Q \subset \mathcal{R}_{g, \ell}$ is irreducible, then $\psi_{R}^{-1}(Q)$ is irreducible in $\mathcal{R}_{g, \ell ; 1}$.

Proof. The fiber of $\psi_{M}$ above the point of $\mathcal{M}_{g}$ representing $X$ is isomorphic to $X$ and is thus irreducible. The fiber of $\psi_{R}$ above the point of $\mathcal{R}_{g, \ell}$ representing $\pi: Y \rightarrow X$ is isomorphic to $Y$ and is thus irreducible. The result follows from Zariski's theorem.

2.4. The $p$-rank. Let $\mu_{p}$ be the kernel of Frobenius on $\mathbb{G}_{m}$. The $p$-rank of a semi-abelian variety $A^{\prime}$ is $f_{A^{\prime}}=\operatorname{dim}_{\mathbb{F}_{p}} \operatorname{Hom}\left(\mu_{p}, A^{\prime}\right)$. If $A^{\prime}$ is an extension of an abelian variety $A$ by a torus $T$, then $f_{A^{\prime}}=f_{A}+\operatorname{rank}(T)$.

For an abelian variety $A$, the $p$-rank can also be defined as the integer $f_{A}$ such that the number of $p$-torsion points in $A(k)$ is $p^{f_{A}}$. If $A$ has dimension $g_{A}$ then $0 \leq f_{A} \leq g_{A}$. The $p$-rank is invariant under isogeny $\sim$ of abelian varieties.

The $p$-rank of a stable curve $X$ is that of $\operatorname{Pic}^{0}(X)$. There are three $p$-ranks associated with an unramified cover $\pi: Y \rightarrow X$, namely the $p$-rank $f$ of $X$, the $p$-rank $f^{\prime}$ of $P_{\pi}$, and the $p$-rank of $Y$ which equals $f+f^{\prime}$.

2.5. The $p$-rank stratification. If $X / S$ is a semi-abelian scheme over a Deligne-Mumford stack, there is a stratification $S=\cup S^{f}$ by locally closed reduced substacks such that $s \in$ $S^{f}(k)$ if and only if $f\left(X_{s}\right)=f$ ([13, Theorem 2.3.1], see also [1, Lemma 2.1]). So, $\mathcal{M}_{g}^{f}$ is the locally closed reduced substack of $\mathcal{M}_{g}$ whose points represent smooth curves of genus $g$ with $p$-rank $f$.

2.6. Compactification of $\mathcal{M}_{g}$. Suppose $X$ is a stable curve with irreducible components $C_{i}$, for $1 \leq i \leq s$. Let $\tilde{C}_{i}$ be the normalization of $C_{i}$. By [3, Example 8, Page 246], $J_{X}$ is canonically an extension of an abelian variety by a torus $T$. There is a short exact sequence:

$$
1 \rightarrow T \rightarrow J_{X} \rightarrow \oplus_{i=1}^{s} J_{\tilde{C}_{i}} \rightarrow 1 .
$$

The rank $r_{T}$ of $T$ is the rank of the cohomology group $H^{1}\left(\Gamma_{X}, \mathbb{Z}\right)$, where $\Gamma_{X}$ denotes the dual graph of $X$. One says that $X$ has compact type if $T$ is trivial.

Let $\overline{\mathcal{M}}_{g}$ be the Deligne-Mumford compactification of $\mathcal{M}_{g}$; it is a smooth proper DeligneMumford stack. The boundary $\partial \mathcal{M}_{g}=\overline{\mathcal{M}}_{g}-\mathcal{M}_{g}$ is the union of the components $\Delta_{0}\left[\overline{\mathcal{M}}_{g}\right]$ and $\Delta_{i}\left[\overline{\mathcal{M}}_{g}\right]$ for $1 \leq i \leq\lfloor g / 2\rfloor$ defined as in [1, Section 2.3].

For $1 \leq i \leq\lfloor g / 2\rfloor$, the $p$-rank $f$ stratum of the boundary component $\Delta_{i}\left[\overline{\mathcal{M}}_{g}\right]$ is the union of the images of the clutching morphisms:

$$
\kappa_{i}: \overline{\mathcal{M}}_{i ; 1}^{f_{1}} \times \overline{\mathcal{M}}_{g-i ; 1}^{f_{2}} \rightarrow \Delta_{i}\left[\overline{\mathcal{M}}_{g}^{f_{1}+f_{2}}\right],
$$


for all pairs $\left\{f_{1}, f_{2}\right\}$ of non-negative integers such that $f_{1}+f_{2}=f$. The $p$-rank $f$ stratum of the boundary component $\Delta_{0}\left[\overline{\mathcal{M}}_{g}\right]$ is the image of the clutching morphism:

$$
\kappa_{0}: \overline{\mathcal{M}}_{g-1 ; 2}^{f-1} \rightarrow \Delta_{0}\left[\overline{\mathcal{M}}_{g}^{f}\right]
$$

\section{The $p$-RANK STRATification of The Boundary of $\mathcal{R}_{g, \ell}$}

In this section, we study Pryms of unramified covers of singular curves. Then we analyze the $p$-rank stratification of the boundary of $\mathcal{R}_{g, \ell}$, whose points typically represent unramified $\mathbb{Z} / \ell$-covers of singular curves. Lemma 3.3 states that every component of the boundary of $\Pi_{\ell}^{-1}\left(\mathcal{M}_{g}^{f}\right)$ has dimension $2 g-4+f$. This is used for Propositions 4.4 and 6.4 . We compute the $p$-rank of the Prym of the cover represented by the generic point of each boundary strata; in particular, Lemma 3.7 is used in Sections 4, 6, and 7.

This section relies on structural results from [6] and [7]. It is necessary to include some material from these references. The following lemma will also be useful.

Lemma 3.1. [22, page 614]. If $A$ and $B$ are substacks of a smooth proper stack $S$ then

$$
\operatorname{codim}(A \cap B, S) \leq \operatorname{codim}(A, S)+\operatorname{codim}(B, S)
$$

3.1. Compactification of $\mathcal{R}_{g, \ell}$. By [7, Definition 1.2], a twisted curve $\mathrm{C}$ is a one dimensional stack such that the corresponding coarse moduli space $C$ is a stable curve whose smooth locus is represented by a scheme and whose singularities are nodes with local picture $\left[\{x y=0\} / \mu_{r}\right]$ with $\zeta \in \mu_{r}$ acting as $\zeta(x, y)=\left(\zeta x, \zeta^{-1} y\right)$. The definition of a faithful line bundle $\eta \in \operatorname{Pic}(\mathrm{C})$ is in [7, Definition 1.3]. By [7, Definition 1.5], a level- $\ell$ twisted curve of genus $g$ is a triple $[\mathrm{X}, \eta, \phi]$ where $\mathrm{X}$ is a twisted curve of genus $g, \eta \in \operatorname{Pic}(\mathrm{X})$ is a faithful line bundle, and $\phi: \eta^{\otimes \ell} \rightarrow \mathcal{O}_{\mathrm{x}}$ is an isomorphism.

By [7, page 6], the moduli space $\mathcal{R}_{g, \ell}$ admits a compactification $\overline{\mathcal{R}}_{g, \ell}$ whose points represent level- $\ell$ twisted curves of genus $g$. It is a smooth Deligne-Mumford stack and there is a finite forgetful morphism $\Pi_{\ell}: \overline{\mathcal{R}}_{g, \ell} \rightarrow \overline{\mathcal{M}}_{g}$.

Let $\partial \mathcal{R}_{g, \ell}=\overline{\mathcal{R}}_{g, \ell}-\mathcal{R}_{g, \ell}$. Some points of $\partial \mathcal{R}_{g, \ell}$ cannot be interpreted in terms of $\ell$-torsion line bundles or $\mathbb{Z} / \ell$-covers of a scheme-theoretic curve. For the sake of intuition, whenever possible, we describe the generic point of a boundary component of $\overline{\mathcal{R}}_{g, \ell}$ in terms of the cover $\pi: Y \rightarrow X$ it represents.

\subsection{Definition of $W_{g}^{f}$.}

Definition 3.2. For $0 \leq f \leq g$, define $W_{g}^{f}=\Pi_{\ell}^{-1}\left(\mathcal{M}_{g}^{f}\right)$ and $\bar{W}_{g}^{f}=\Pi_{\ell}^{-1}\left(\overline{\mathcal{M}}_{g}^{f}\right)$.

The points of $W_{g}^{f}$ represent unramified $\mathbb{Z} / \ell$-covers $\pi: Y \rightarrow X$ of a smooth curve $X$ of genus $g$ and $p$-rank $f$.

Lemma 3.3. Let $g \geq 1$ and $0 \leq f \leq g$. Then $W_{g}^{f}$ is non-empty. For $g \geq 2$, let $Q$ be an irreducible component of $\bar{W}_{g}^{f}$. Then

(1) $Q$ has dimension $2 g-3+f$;

(2) $W_{g}^{f} \cap Q$ is open and dense in $Q$ (the generic point of $Q$ represents a smooth curve);

(3) the dimension of every component of $Q \cap \partial \mathcal{R}_{g, \ell}$ is $2 g-4+f$.

Proof. Since $\Pi_{\ell}: \overline{\mathcal{R}}_{g, \ell} \rightarrow \overline{\mathcal{M}}_{g}$ is finite, flat, and surjective, these facts follow from the analogous facts for $\overline{\mathcal{M}}_{g}^{f}$; see [9, Theorem 2.3] for part (1) and [1, Lemmas 3.1, 3.2(a)] for parts (2) - (3). 
3.3. Boundary components of $\overline{\mathcal{R}}_{g, \ell}$. Let $\partial \mathcal{R}_{g, \ell}=\overline{\mathcal{R}}_{g, \ell}-\mathcal{R}_{g, \ell}$ denote the boundary of $\mathcal{R}_{g, \ell}$. Informally, the points of $\partial \mathcal{R}_{g, \ell}$ represent unramified $\mathbb{Z} / \ell$-covers of singular curves, although we make this more precise below. For covers of singular curves of compact type, the boundary components lie above $\Delta_{i}\left[\overline{\mathcal{M}}_{g}\right]$ for some $1 \leq i \leq\lfloor g / 2\rfloor$ and are denoted $\Delta_{i: g-i}$, $\Delta_{i}$, and $\Delta_{g-i}$. For covers of singular curves of non-compact type, the boundary components lie above $\Delta_{0}\left[\overline{\mathcal{M}}_{g}\right]$ and are denoted $\Delta_{0, I}, \Delta_{0, I I}$ and $\Delta_{0, I I I}^{(a)}$.

In Sections 3.4 and 3.5, we recall the definition of these boundary components and investigate them in terms of the $p$-rank. Before doing this, recall the following results.

Proposition 3.4. [6, Equation (16)] For $1 \leq i<\lfloor g / 2\rfloor$, there is an equality of divisors

$$
\Pi_{\ell}^{*}\left(\Delta_{i}\left[\overline{\mathcal{M}}_{g}\right]\right)=\Delta_{i}\left[\overline{\mathcal{R}}_{g, \ell}\right]+\Delta_{g-i}\left[\overline{\mathcal{R}}_{g, \ell}\right]+\Delta_{i: g-i}\left[\overline{\mathcal{R}}_{g, \ell}\right] .
$$

If $g$ is even, there is an equality of divisors

$$
\Pi_{\ell}^{*}\left(\Delta_{g / 2}\left[\overline{\mathcal{M}}_{g}\right]\right)=\Delta_{g / 2}\left[\overline{\mathcal{R}}_{g, \ell}\right]+\Delta_{g / 2: g / 2}\left[\overline{\mathcal{R}}_{g, \ell}\right] .
$$

Proposition 3.5. [6, page 89] or [7, Equation (17)] There is an equality of divisors

$$
\Pi_{\ell}^{*}\left(\Delta_{0}\left[\overline{\mathcal{M}}_{g}\right]\right)=\Delta_{0, I}\left[\overline{\mathcal{R}}_{g, \ell}\right]+\Delta_{0, I I}\left[\overline{\mathcal{R}}_{g, \ell}\right]+\ell \sum_{a=0}^{\ell \ell / 2\rfloor} \Delta_{0, I I I}^{(a)}\left[\overline{\mathcal{R}}_{g, \ell}\right] .
$$

3.4. Pryms of covers of singular curves of compact type. Let $X$ be a singular curve formed by intersecting two curves $C_{1}$ and $C_{2}$ (at points $x_{1} \in C_{1}$ and $x_{2} \in C_{2}$ ) in an ordinary double point. By (1), $J_{X} \simeq J_{C_{1}} \oplus J_{C_{2}}$. Let $\xi$ be the point of $\Delta_{i}\left[\overline{\mathcal{M}}_{g}\right]$ representing $X$. Then an unramified cyclic degree $\ell$ cover $\pi: Y \rightarrow X$ is determined by two line bundles $\eta_{C_{1}} \in \operatorname{Pic}^{0}\left(C_{1}\right)[\ell]$ and $\eta_{C_{2}} \in \operatorname{Pic}^{0}\left(C_{2}\right)[\ell]$, which are not both trivial. The points of $\Delta_{i: g-i}\left[\overline{\mathcal{R}}_{g, \ell}\right]$ above $\xi$ represent covers $\pi$ for which both $\eta_{C_{1}}$ and $\eta_{C_{2}}$ are nontrivial; the points of $\Delta_{i}\left[\overline{\mathcal{R}}_{g, \ell}\right]$ (resp. $\Delta_{g-i}\left[\overline{\mathcal{R}}_{g, \ell}\right]$ ) above $\xi$ represent covers $\pi$ for which $\eta_{C_{1}}$ (resp. $\eta_{C_{2}}$ ) is trivial.

3.4.1. The boundary component $\Delta_{i: g-i}$. The boundary divisor $\Delta_{i: g-i}\left[\overline{\mathcal{R}}_{g, \ell}\right]$ is the image of the clutching map

$$
\kappa_{i: g-i}: \overline{\mathcal{R}}_{i, \ell ; 1} \times \overline{\mathcal{R}}_{g-i, \ell ; 1} \rightarrow \overline{\mathcal{R}}_{g, \ell}
$$

defined on a generic point as follows. Let $\tau_{1}$ be a point of $\overline{\mathcal{R}}_{i, \ell ; 1}$ representing $\left(\pi_{1}: C_{1}^{\prime} \rightarrow\right.$ $\left.C_{1}, x_{1}^{\prime} \mapsto x_{1}\right)$ and let $\tau_{2}$ be a point of $\overline{\mathcal{R}}_{g-i, \ell ; 1}$ representing $\left(\pi_{2}: C_{2}^{\prime} \rightarrow C_{2}, x_{2}^{\prime} \mapsto x_{2}\right)$. Let $Y$ be the curve with components $C_{1}^{\prime}$ and $C_{2}^{\prime}$, formed by identifying $\sigma^{k}\left(x_{1}^{\prime}\right)$ and $\sigma^{k}\left(x_{2}^{\prime}\right)$ in an ordinary double point for $0 \leq k \leq \ell-1$. Then $\kappa_{i: g-i}\left(\tau_{1}, \tau_{2}\right)$ is the point representing the unramified $\mathbb{Z} / \ell$-cover $Y \rightarrow X$. This is illustrated in Figure 1 for $\ell=2$.

Lemma 3.6. The clutching map $\kappa_{i: g-i}$ restricts to a map

$$
\kappa_{i: g-i}: \bar{W}_{i ; 1}^{f_{1}} \times \bar{W}_{g-i ; 1}^{f_{2}} \rightarrow \Delta_{i: g-i}\left[\bar{W}_{g}^{f_{1}+f_{2}}\right] .
$$

Proof. This follows from (2).

Lemma 3.7. Suppose $\pi: Y \rightarrow X$ is an unramified $\mathbb{Z} / \ell$-cover represented by a point of $\Delta_{i: g-i}\left[\overline{\mathcal{R}}_{g, \ell}\right]$. Then $P_{\pi}$ is an extension of a semi-abelian variety $P_{\pi_{1}} \oplus P_{\pi_{2}}$ by a torus $T$ of rank $r_{T}=\ell-1$. If $f_{i}^{\prime}$ is the $p$-rank of $P_{\pi_{i}}$, then the p-rank of $P_{\pi}$ is $f_{1}^{\prime}+f_{2}^{\prime}+(\ell-1)$.

Proof. By (1), $J_{Y}$ is an extension of $J_{C_{1}^{\prime}} \oplus J_{C_{2}^{\prime}}$ by a torus $T$ whose rank $r_{T}$ is the rank of $H^{1}\left(\Gamma_{Y}, \mathbb{Z}\right)$. Then $r_{T}=\ell-1$ since $\Gamma_{Y}$ consists of two vertices, for the two irreducible 


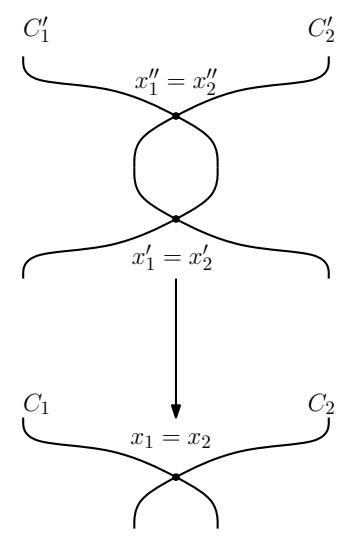

FiguRE $1 . \Delta_{i: g-i}: \eta_{C_{1}} ¥ \mathcal{O}_{C_{1}}, \eta_{C_{2}} ¥ \mathcal{O}_{C_{2}}$

components $C_{1}^{\prime}, C_{2}^{\prime}$, which are connected with $\ell$ edges, corresponding to the $\ell$ intersection points. There is a commutative diagram with exact rows

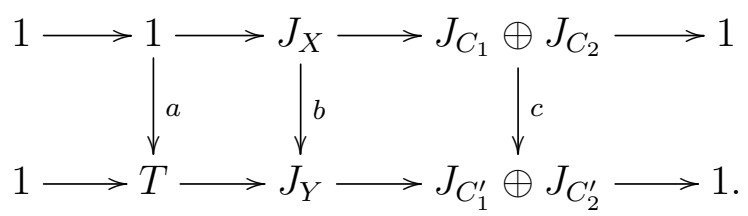

By the snake lemma, there is an exact sequence

$$
1 \rightarrow \operatorname{Coker}(a) \rightarrow \operatorname{Coker}(b) \rightarrow \operatorname{Coker}(c) \rightarrow 1,
$$

and thus an exact sequence

$$
1 \rightarrow T \rightarrow P_{\pi} \rightarrow P_{\pi_{1}} \oplus P_{\pi_{2}} \rightarrow 1
$$

From (4), $f_{P_{\pi}}=f_{P_{\pi_{1}} \oplus P_{\pi_{2}}}+\operatorname{rank}(T)=f_{1}^{\prime}+f_{2}^{\prime}+(\ell-1)$.

3.4.2. The boundary component $\Delta_{i}, i>0$ : The boundary divisor $\Delta_{i}\left[\overline{\mathcal{R}}_{g}\right]$ is the image of the clutching map

$$
\kappa_{i}: \overline{\mathcal{R}}_{i, \ell ; 1} \times \overline{\mathcal{M}}_{g-i ; 1} \rightarrow \overline{\mathcal{R}}_{g, \ell},
$$

defined on a generic point as follows. Let $\tau$ be a point of $\mathcal{R}_{i, \ell ; 1}$ representing $\left(\pi_{1}^{\prime}: C_{1}^{\prime} \rightarrow\right.$ $\left.C_{1}, x^{\prime} \mapsto x\right)$ and let $\omega$ be a point of $\mathcal{M}_{g-i ; 1}$ representing $\left(C_{2}, x_{2}\right)$. Let $Y$ be the curve with components $C_{1}^{\prime}$ and $\ell$ copies of $C_{2}$, formed by identifying the point $x_{2}$ on each copy of $C_{2}$ with a point of $\pi_{1}^{-1}\left(x_{1}\right)$. Then $\kappa_{i}(\tau, \omega)$ represents the unramified $\mathbb{Z} / \ell$-cover $\pi: Y \rightarrow X$. See Figure 2 for $\ell=2$.

Lemma 3.8. The clutching map $\kappa_{i}$ restricts to a map:

$$
\kappa_{i}: \bar{W}_{i ; 1}^{f_{1}} \times \overline{\mathcal{M}}_{g-i ; 1}^{f_{2}} \rightarrow \Delta_{i}\left[\bar{W}_{g}^{f_{1}+f_{2}}\right] .
$$

Proof. This follows from (2).

Lemma 3.9. Suppose $\pi: Y \rightarrow X$ is an unramified $\mathbb{Z} / \ell$-cover represented by a point of $\Delta_{i}\left[\overline{\mathcal{R}}_{g, \ell}\right]$. Then $P_{\pi} \simeq P_{\pi_{1}} \oplus J_{C_{2}}^{\ell-1}$. If $f_{1}^{\prime}$ is the p-rank of $P_{\pi_{1}}$, then the p-rank of $P_{\pi}$ is $f_{1}^{\prime}+(\ell-1) f_{2}$.

Proof. By construction, $J_{Y} \simeq J_{C_{1}^{\prime}} \oplus J_{C_{2}}^{\ell}$. The image of $(1-\sigma)$ on $J_{Y}$ is $P_{\pi}$, while on $J_{C_{1}^{\prime}} \oplus J_{C_{2}}^{\ell}$ it is $P_{\pi_{1}} \oplus J_{C_{2}}^{\ell-1}$. Then the $p$-rank is the sum of the $p$-ranks of $P_{\pi_{1}}$ and $J_{C_{2}}^{\ell-1}$. 


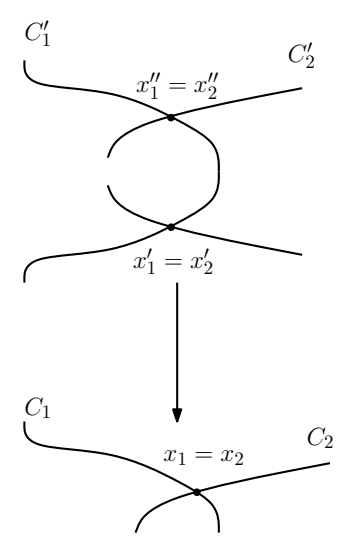

FiguRE 2. $\Delta_{i}: \eta_{C_{1}} \not \mathcal{O}_{C_{1}}, \eta_{C_{2}} \simeq \mathcal{O}_{C_{2}}$

3.5. Pryms of covers of singular curves of non-compact type. This material is needed only for future work. The main reference is [10, Example 6.5] when $\ell=2$ and [6, Section 1.4] and [7, Section 1.5.2] for general $\ell$. Let $\left(X^{\prime}, x, y\right)$ be a curve of genus $g-1$ with 2 marked points. Let $X$ be a curve of genus $g$ of non-compact type formed by identifying two points $x, y$ on $X^{\prime}$. By (1), if $X^{\prime}$ has $p$-rank $f_{1}$, then $X$ has $p$-rank $f=f_{1}+1$.

3.5.1. The Boundary Component $\Delta_{0, I}$. The boundary divisor $\Delta_{0, I}\left[\overline{\mathcal{R}}_{g, \ell}\right]$ is the image of the clutching map

$$
\kappa_{0, I}: \overline{\mathcal{R}}_{g-1, \ell ; 2} \rightarrow \overline{\mathcal{R}}_{g, \ell}
$$

defined on a generic point as follows. Let $\tau$ be a point of $\mathcal{R}_{g-1, \ell ; 2}$ representing $\left(\pi^{\prime}: Y^{\prime} \rightarrow\right.$ $X^{\prime}, x^{\prime} \mapsto x, y^{\prime} \mapsto y$ ) (two markings). Let $Y$ be the nodal curve of non-compact type with normalization $Y^{\prime}$, formed by identifying $\sigma^{k}\left(x^{\prime}\right)$ and $\sigma^{k}\left(y^{\prime}\right)$, for $0 \leq k \leq \ell-1$, in an ordinary double point. Then $\kappa_{0, I}(\tau)$ is the point representing the unramified $\mathbb{Z} / \ell$-cover $\pi: Y \rightarrow X$. See Figure 3 for the case $\ell=2$.

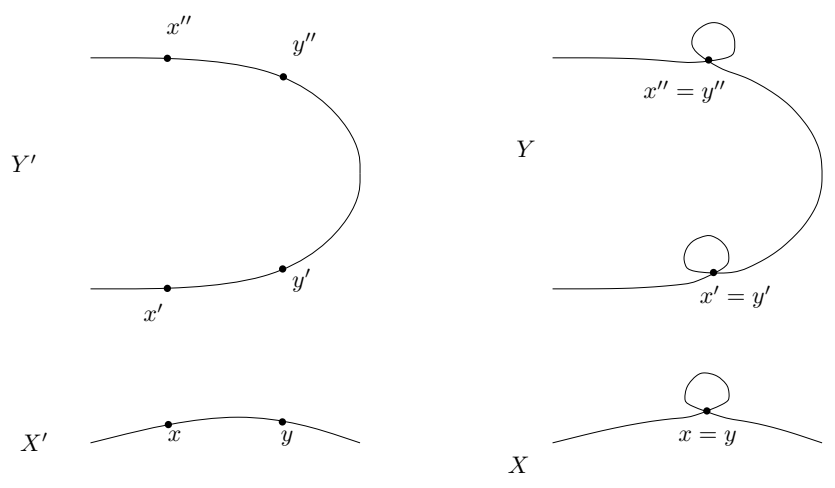

FiguRE $3 . \Delta_{0, I}, \ell=2$

Lemma 3.10. The clutching map $\kappa_{0, I}$ restricts to a map: $\kappa_{0, I}: \bar{W}_{g-1 ; 2}^{f_{1}} \rightarrow \Delta_{0, I}\left[\bar{W}_{g}^{f_{1}+1}\right]$.

Proof. This follows from (3).

Lemma 3.11. Suppose $\pi: Y \rightarrow X$ is an unramified $\mathbb{Z} / \ell$-cover represented by a point of $\Delta_{0, I}\left[\overline{\mathcal{R}}_{g, \ell}\right]$. Then $P_{\pi}$ is an extension of a semi-abelian variety $P_{\pi^{\prime}}$ by a torus $T$ with $r_{T}=\ell-1$. If $f_{1}^{\prime}$ is the $p$-rank of $P_{\pi^{\prime}}$, then the $p$-rank of $P_{\pi}$ is $f^{\prime}=f_{1}^{\prime}+(\ell-1)$. 
Proof. There is a commutative diagram with exact rows:

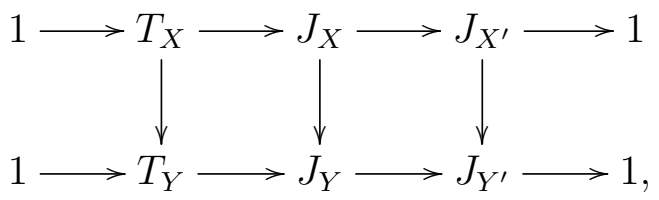

where $T_{X}$ is a torus of rank 1 and $T_{Y}$ is a torus of rank $\ell$. Then $T_{Y} / T_{X}$ is a torus $T$ of rank $\ell-1$. By the snake lemma, there is an exact sequence $1 \rightarrow T \rightarrow P_{\pi} \rightarrow P_{\pi^{\prime}} \rightarrow 1$.

3.5.2. The Boundary Component $\Delta_{0, I I}$. The boundary divisor $\Delta_{0, I I}\left[\overline{\mathcal{R}}_{g, \ell}\right]$ is the image of the clutching map

$$
\kappa_{0, I I}: \overline{\mathcal{M}}_{g-1 ; 2} \rightarrow \overline{\mathcal{R}}_{g, \ell}
$$

defined on a generic point as follows. Let $\omega$ be a point of $\overline{\mathcal{M}}_{g-1 ; 2}$ representing $\left(X^{\prime}, x, y\right)$ (with 2 markings). Consider a disconnected curve with components $\left(X_{i}^{\prime}, x_{i}, y_{i}\right)$ indexed by $i \in \mathbb{Z} / \ell$ such that each component is isomorphic to $\left(X^{\prime}, x, y\right)$.

Let $Y$ be the nodal curve of non-compact type formed by identifying $\sigma^{k}\left(x_{1}\right)$ and $\sigma^{k+1}\left(y_{1}\right)$, for $0 \leq k \leq \ell-1$, in an ordinary double point. Then $\kappa_{0, I I}(\omega)$ is the point representing the $\mathbb{Z} / \ell$-cover $\pi: Y \rightarrow C$. This is illustrated in Figure 4 for $\ell=2$.
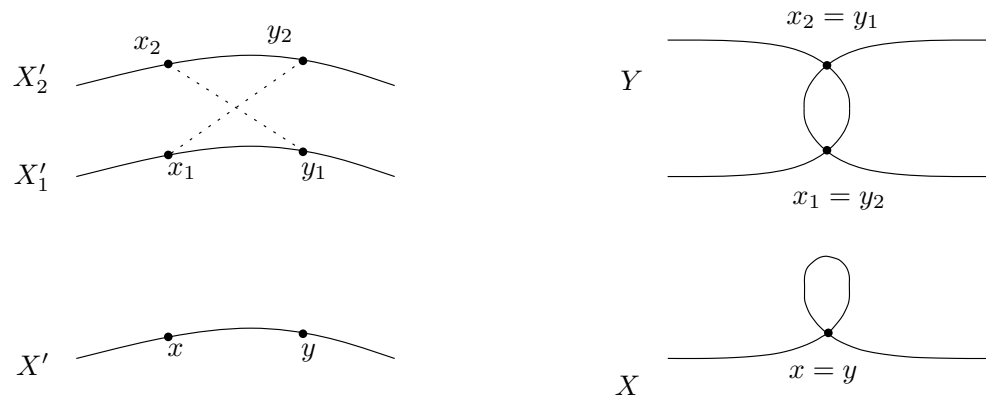

FigURE $4 . \Delta_{0, I I, \ell=2}$

Lemma 3.12. The clutching map $\kappa_{0, I I}$ restricts to a map $\kappa_{0, I I}: \overline{\mathcal{M}}_{g-1 ; 2}^{f_{1}} \rightarrow \Delta_{0, I I}\left[\bar{W}_{g}^{f_{1}+1}\right]$.

Proof. This follows from (3).

Lemma 3.13. Suppose $\pi: Y \rightarrow X$ is an unramified $\mathbb{Z} / \ell$-cover represented by a point of $\Delta_{0, I}\left[\overline{\mathcal{R}}_{g, \ell}\right]$. Then $P_{\pi} \simeq J_{X^{\prime}}^{\ell-1}$. If $f_{1}$ is the $p$-rank of $X^{\prime}$, then the $p$-rank of $P_{\pi}$ is $(\ell-1) f_{1}$.

Proof. Like Lemma 3.11, but $T_{X}$ and $T_{Y}$ have rank 1 .

3.5.3. The Boundary Component $\Delta_{0, I I I}$. The points of the last boundary component(s) represent level- $\ell$ twisted curves $[X, \tilde{\eta}, \tilde{\phi}]$ which have the following structure. Let $\left(X^{\prime}, x, y\right)$ be a point of $\overline{\mathcal{M}}_{g-1 ; 2}$ and let $E$ be a projective line. The curve $X=X^{\prime} \cup_{x, y} E$ of genus $g$ has components $X^{\prime}$ and $E$, with two ordinary double points formed by identifying $x$ with 0 and $y$ with $\infty$. Since $E$ is an exceptional component, the restriction of $\tilde{\eta}$ to $E$ is $\eta_{E}=\mathcal{O}_{E}(1)$. This implies that the restriction $\eta_{X^{\prime}}$ of $\tilde{\eta}$ to $X^{\prime}$ has degree -1 and that $\eta_{X^{\prime}}^{\otimes-\ell}=\mathcal{O}_{X^{\prime}}(a x+(\ell-a) y)$, for some $1 \leq a \leq \ell-1$.

The boundary divisor $\Delta_{0, I I I}^{(a)}$ is the closure in $\overline{\mathcal{R}}_{g-1, \ell}$ of points representing such level- $\ell$ twisted curves $[X, \tilde{\eta}, \tilde{\phi}]$. For complete details, see [6, Section 1.4]. There is a clutching map

$$
\kappa_{0, I I I}^{(a)}: \bar{M}_{g-1 ; 2} \rightarrow \Delta_{0, I I I}^{(a)}\left[\bar{R}_{g, \ell}\right] .
$$


Lemma 3.14. The clutching map $\kappa_{0, I I I}^{(a)}$ restricts to a map

$$
\kappa_{0, I I I}^{(a)}: \bar{W}_{g-1 ; 2}^{f_{1}} \rightarrow \Delta_{0, I I I}^{(a)}\left[\bar{W}_{g}^{f_{1}+1}\right] .
$$

Proof. This follows from (3).

\section{Ordinary Pryms of a Generic CURVE of GIVEn $p$-RANK}

The main result of this section is that the Prym variety of an unramified $\mathbb{Z} / \ell$-cover of a generic curve of genus $g$ and $p$-rank $f$ is ordinary, Theorem 4.5. We prove this using degeneration to $\partial \mathcal{R}_{g, \ell}$ and information about the $\mathbb{Z} / \ell$-monodromy of components of $\mathcal{M}_{g}^{f}$. The monodromy results are needed since all curves represented by a point of $\Delta_{i}\left[\overline{\mathcal{M}}_{g}^{f}\right]$ have an unramified $\mathbb{Z} / \ell$-cover with non-ordinary Prym, when $f<g$ and $0 \leq i<g$, as seen in Sections 3.4 .2 and 3.5 .

\subsection{Earlier work on the $p$-rank stratification of $\mathcal{M}_{g}$.}

Proposition 4.1. [1, Proposition 3.4] Let $g \geq 2$ and $0 \leq f \leq g$. Suppose $1 \leq i \leq g-1$ and $\left(f_{1}, f_{2}\right)$ is a pair such that $f_{1}+f_{2}=f$ and $0 \leq f_{1} \leq i$ and $0 \leq f_{2} \leq g-i$. Let $S$ be an irreducible component of $\mathcal{M}_{g}^{f}$ and let $\bar{S}$ be its closure in $\overline{\mathcal{M}}_{g}$.

(1) Then $\bar{S}$ intersects $\kappa_{i: g-i}\left(\overline{\mathcal{M}}_{i ; 1}^{f_{1}} \times \overline{\mathcal{M}}_{g-i ; 1}^{f_{2}}\right)$.

(2) Each irreducible component of the intersection contains the image of a component of $\overline{\mathcal{M}}_{i ; 1}^{f_{1}} \times \overline{\mathcal{M}}_{g-i ; 1}^{f_{2}}$.

Let $\mathcal{C} \rightarrow S$ be a relative proper semi-stable curve of compact type of genus $g$ over $S$. Then $\operatorname{Pic}^{0}(\mathcal{C})[\ell]$ is an étale cover of $S$ with geometric fiber isomorphic to $(\mathbb{Z} / \ell)^{2 g}$. For each $n \in \mathbb{N}$, the fundamental group $\pi_{1}(S, s)$ acts linearly on the fiber $\operatorname{Pic}^{0}(\mathcal{C})\left[\ell^{n}\right]_{s}$, and the monodromy group $\mathrm{M}_{\ell^{n}}(\mathcal{C} \rightarrow S, s)$ is the image of $\pi_{1}(S, s)$ in $\operatorname{Aut}\left(\operatorname{Pic}^{0}(\mathcal{C})\left[\ell^{n}\right]_{s}\right)$. Also $\mathrm{M}_{\mathbb{Z}_{\ell}}(S, s):=\lim _{\overleftarrow{n}} \mathrm{M}_{\ell^{n}}(S, s)$ is the $\ell$-adic monodromy group. When $S$ is an irreducible component of $\mathcal{M}_{g}^{f}$ and $\mathcal{C} \rightarrow S$ is the tautological curve, the next result states that $\mathrm{M}_{\ell}(S):=\mathrm{M}_{\ell}(\mathcal{C} \rightarrow S, s)$ and $\mathrm{M}_{\mathbb{Z}_{\ell}}(S):=\mathrm{M}_{\mathbb{Z}_{\ell}}(\mathcal{C} \rightarrow S, s)$ are as large as possible.

Theorem 4.2. [1, Theorem 4.5] Let $\ell$ be a prime distinct from $p$; let $g \geq 2$ and $0 \leq f \leq g$ with $f \neq 0$ if $g=2$. Let $S$ be an irreducible component of $\mathcal{M}_{g}^{f}$, the p-rank $f$ stratum in $\mathcal{M}_{g}$. Then $\mathrm{M}_{\ell}(S) \simeq \operatorname{Sp}_{2 g}(\mathbb{Z} / \ell)$ and $\mathrm{M}_{\mathbb{Z}_{\ell}}(S) \simeq \operatorname{Sp}_{2 g}\left(\mathbb{Z}_{\ell}\right)$.

4.2. Irreducibility of fibers of $\Pi_{\ell}$ over $\mathcal{M}_{g}^{f}$. Recall that the morphism $\Pi_{\ell}: \mathcal{R}_{g, \ell} \rightarrow \mathcal{M}_{g}$, which sends the point representing the cover $\pi: Y \rightarrow X$ to the point representing the curve $X$, is finite and flat with degree $\ell^{2 g}-1$.

Proposition 4.3. Under the hypotheses of Theorem 4.2, if $S$ is an irreducible component of $\mathcal{M}_{g}^{f}$, then $\Pi_{\ell}^{-1}(S)$ is irreducible.

Proof. Equip $(\mathbb{Z} / \ell)^{2 g}$ with the standard symplectic pairing $\langle\cdot, \cdot\rangle_{\text {std }}$, and let

$$
S_{[\ell]}:=\operatorname{Isom}\left(\left(\operatorname{Pic}^{0}(\mathcal{C} / S)[\ell],\langle\cdot, \cdot\rangle_{\lambda}\right),\left((\mathbb{Z} / \ell)_{S}^{2 g},\langle\cdot, \cdot\rangle_{\text {std }}\right)\right) .
$$

There is an $\ell$ th root of unity on $S$, so $S_{[\ell]} \rightarrow S$ is an étale Galois cover, possibly disconnected, with covering group $\operatorname{Sp}_{2 g}(\mathbb{Z} / \ell)$. By Theorem 4.2. $\mathrm{M}_{\ell}(S) \simeq \operatorname{Sp}_{2 \mathrm{~g}}(\mathbb{Z} / \ell)$. The geometric interpretation of this is that $S_{[\ell]}$ is irreducible.

Suppose $\tilde{\xi}$ is a point of $S_{[\ell]}$. Then $\tilde{\xi}$ represents a curve $X$, together with an isomorphism between $(\mathbb{Z} / \ell)^{2 g}$ and $\operatorname{Pic}^{0}(X)[\ell]$. The isomorphism identifies $(1,0, \ldots, 0)$ with a point of 
order $\ell$ on $J_{X}$. It follows that $\tilde{\xi}$ determines an unramified $\mathbb{Z} / \ell$-cover $\pi: Y \rightarrow X$. Thus there is a forgetful morphism $F: S_{[\ell]} \rightarrow \Pi_{\ell}^{-1}(S)$. Then $\Pi_{\ell}^{-1}(S)$ is irreducible because $S_{[\ell]}$ is.

\subsection{Key degeneration result.}

Proposition 4.4. Let $g \geq 3$ and $0 \leq f \leq g$. Let $Q$ be an irreducible component of $\bar{W}_{g}^{f}$.

(1) Then $Q$ intersects $\Delta_{i: g-i}$ for each $1 \leq i \leq\lfloor g / 2\rfloor$.

(2) More generally, if $\left(f_{1}, f_{2}\right)$ is a pair such that $f_{1}+f_{2}=f$ and $0 \leq f_{1} \leq i$ and $0 \leq f_{2} \leq g-i$, then $Q$ contains the image of a component of $\kappa_{i ; g-i}\left(\bar{W}_{i ; 1}^{f_{1}} \times \bar{W}_{g-i ; 1}^{f_{2}}\right)$.

Proof. By Proposition 4.3, $Q=\Pi_{\ell}^{-1}(S)$ for some irreducible component $S$ of $\overline{\mathcal{M}}_{g}^{f}$. By Proposition 4.1. $S$ contains the image of a component of $\overline{\mathcal{M}}_{i ; 1}^{f_{1}} \times \overline{\mathcal{M}}_{g-i ; 1}^{f_{2}}$. Consider a point $\xi$ of $Q$ lying above this image. Then $\xi$ represents an unramified $\mathbb{Z} / \ell$-cover $\pi: Y \rightarrow X$ as in Section 3.4.1. By definition, $X$ is a stable curve having components $C_{1}$ and $C_{2}$ of genera $i$ and $g-i$ and $p$-ranks $f_{1}$ and $f_{2}$.

The $\mathbb{Z} / \ell$-cover $\pi$ is determined by a point of order $\ell$ on $J_{X}$. Now $J_{X} \simeq J_{C_{1}} \oplus J_{C_{2}}$, so $J_{X}[\ell] \simeq J_{C_{1}}[\ell] \oplus J_{C_{2}}[\ell]$. The point $\xi$ is in $\Delta_{i}$ or $\Delta_{g-i}$ if and only if the point of order $\ell$ is in either $J_{C_{1}}[\ell] \oplus\{0\}$ or $\{0\} \oplus J_{C_{2}}[\ell]$. There are $\ell^{2 g}-\ell^{2 i}-\ell^{2(g-i)}+1$ points of order $\ell$ which do not have this property. Since $Q=\Pi_{\ell}^{-1}(S)$, without loss of generality, one can suppose that the point of order $\ell$ is one of these or, equivalently, that $\xi$ is in $\Delta_{i: g-i}$, completing part (1).

Every component of $Q \cap \Delta_{i: g-i}$ has dimension $2 g-4+f$. By Lemma 3.3(3), this equals the dimension of the components of $\kappa_{i: g-i}\left(\bar{W}_{i ; 1}^{f_{1}} \times \bar{W}_{g-i ; 1}^{f_{2}}\right)$, finishing part (2).

4.4. Ordinary Pryms. The first theorem is that the Prym of an unramified $\mathbb{Z} / \ell$-cover of a generic curve of genus $g$ and $p$-rank $f$ is ordinary, for any $0 \leq f \leq g$.

Theorem 4.5. Let $\ell$ be a prime distinct from $p$; let $g \geq 2$ and $0 \leq f \leq g$ with $f \neq 0$ if $g=2$. If $Q$ is an irreducible component of $W_{g}^{f}=\Pi_{\ell}^{-1}\left(\mathcal{M}_{g}^{f}\right)$, then the Prym of the cover represented by the generic point of $Q$ is ordinary (with p-rank $f_{Q}^{\prime}=(\ell-1)(g-1)$ ).

Proof. The proof is by induction on $g$, with the base case $g=1$ being vacuous. Suppose the result is true for all $1 \leq g^{\prime}<g$. Let $Q$ be an irreducible component of $W_{g}^{f}$. Let $\bar{Q}$ be its closure in $\overline{\mathcal{R}}_{g, \ell}$. Choose $i$ such that $1 \leq i \leq g-1$ and a pair $\left(f_{1}, f_{2}\right)$ such that $f_{1}+f_{2}=f$ and $0 \leq f_{1} \leq i$ and $0 \leq f_{2} \leq g-i$. Note that one can avoid the choice $i=2$ and $f_{1}=0$. By Proposition 4.4 . $\bar{Q}$ contains a component of $\kappa_{i: g-i}\left(\bar{W}_{i, 1}^{f_{1}} \times \bar{W}_{g-i, 1}^{f_{2}}\right)$.

Let $f_{Q}^{\prime}, f_{\partial Q}^{\prime}, f_{1}^{\prime}$, and $f_{2}^{\prime}$ respectively denote the $p$-rank of the Prym of the cover represented by the generic point of a component of $Q, \partial Q, \bar{W}_{i ; 1}^{f_{1}}$ and $\bar{W}_{g-i ; 1}^{f_{2}}$. By semi-continuity $f_{Q}^{\prime} \geq f_{\partial Q}^{\prime}$. By Lemma 3.7. $f_{\partial Q}^{\prime}=f_{1}^{\prime}+f_{2}^{\prime}+(\ell-1)$. By the inductive hypothesis, $f_{1}^{\prime}=(\ell-1)(i-1)$ and $f_{2}^{\prime}=(\ell-1)(g-i-1)$. Thus $f_{Q}^{\prime} \geq(\ell-1)(g-1)$ which equals $\operatorname{dim}\left(P_{\pi}\right)$.

Theorem 1.1(1) follows from Proposition 4.3 and Theorem 4.5.

\section{Purity Results}

5.1. A stratification of $\overline{\mathcal{R}}_{g}$ by the $p$-ranks of $X$ and $P_{\pi}$. When $\ell=2$ and $p$ is odd, we consider the stratification of $\mathcal{R}_{g, 2}$ by $p$-rank. Proposition 5.2 gives a lower bound for the dimension of the $p$-rank strata. Since this section is only about double covers, the subscript $\ell=2$ is dropped from the notation for simplicity. 
If $\pi: Y \rightarrow X$ is an unramified double cover, let $f^{\prime}$ denote the $p$-rank of $P_{\pi}$. Let $\tilde{\mathcal{A}}_{g-1}^{f^{\prime}}$ denote the $p$-rank $f^{\prime}$ stratum of the toroidal compactification $\tilde{\mathcal{A}}_{g-1}$ of the moduli space $\mathcal{A}_{g-1}$ of principally polarized abelian varieties of dimension $g-1$. The Prym map $\operatorname{Pr}_{g}: \overline{\mathcal{R}}_{g} \rightarrow \tilde{\mathcal{A}}_{g-1}$ sends the point representing $\pi: Y \rightarrow X$ to the point representing the principally polarized abelian variety $P_{\pi}$. The image and fibers of $P r_{g}$ are well understood only for $2 \leq g \leq 6$.

Definition 5.1. Let $0 \leq f \leq g$ and $0 \leq f^{\prime} \leq g-1$. Define $\bar{V}_{g}^{f^{\prime}}=\operatorname{Pr}_{g}^{-1}\left(\tilde{\mathcal{A}}_{g-1}^{f^{\prime}}\right)$ and $V_{g}^{f^{\prime}}=\bar{V}_{g}^{f^{\prime}} \cap \mathcal{R}_{g}$. Define $\overline{\mathcal{R}}_{g}^{\left(f, f^{\prime}\right)}=\bar{W}_{g}^{f} \cap \bar{V}_{g}^{f^{\prime}}$ and $\mathcal{R}_{g}^{\left(f, f^{\prime}\right)}=\overline{\mathcal{R}}_{g}^{\left(f, f^{\prime}\right)} \cap \mathcal{R}_{g}$.

Hence, the points of $V_{g}^{f^{\prime}}\left(\right.$ resp. $\mathcal{R}_{g}^{\left(f, f^{\prime}\right)}$ ) represent unramified double covers $\pi: Y \rightarrow X$ of a smooth curve $X$ of genus $g$ (resp. and $p$-rank $f$ ) such that $P_{\pi}$ has $p$-rank $f^{\prime}$.

By Theorem 4.5. if $p \geq 3$, then $\mathcal{R}_{g}^{f, g-1}$ is non-empty of dimension $2 g-3+f$ for all $g \geq 2$ and $0 \leq f \leq g$ with $f \neq 0$ if $g=2$. Applying purity yields the following result.

Proposition 5.2. Let $\ell=2, p \geq 3, g \geq 2$ and $0 \leq f \leq g$. For $0 \leq f^{\prime} \leq g-2$, if $\mathcal{R}_{g}^{\left(f, f^{\prime}\right)}$ (resp. $\left.\overline{\mathcal{R}}_{g}^{\left(f, f^{\prime}\right)}\right)$ is non-empty, then each of its components has dimension at least $g-2+\left(f+f^{\prime}\right)$.

Proof. Consider the forgetful morphism $\tau_{g}: \mathcal{R}_{g} \rightarrow \mathcal{M}_{2 g-1}$ which sends the point representing $\pi: Y \rightarrow X$ to the point representing $Y$. If $g \geq 2$, then the genus of $Y$ is at least 3 and $\operatorname{Aut}_{k}(Y)$ is finite. So $\tau_{g}$ is finite-to- 1 and its image has dimension $3 g-3$.

Let $U$ be a component of $\mathcal{R}_{g}^{\left(f, f^{\prime}\right)}$ and let $Z$ be its image under $\tau_{g}$. If $\pi: Y \rightarrow X$ is represented by a point of $U$, then the $p$-rank of $Y$ is $f+f^{\prime}$. Thus $Z$ is contained in $\mathcal{M}_{2 g-1}^{f+f^{\prime}}$. Note that $Z$ is a component of $\operatorname{Im}\left(\tau_{g}\right) \cap \mathcal{M}_{2 g-1}^{f+f^{\prime}}$. By Lemma 3.1.

$$
\operatorname{codim}\left(\operatorname{Im}\left(\tau_{g}\right) \cap \mathcal{M}_{2 g-1}^{f+f^{\prime}}, \operatorname{Im}\left(\tau_{g}\right)\right) \leq \operatorname{codim}\left(\mathcal{M}_{2 g-1}^{f+f^{\prime}}, \mathcal{M}_{2 g-1}\right) .
$$

By [9, Theorem 2.3], $\operatorname{codim}\left(\mathcal{M}_{2 g-1}^{f+f^{\prime}}, \mathcal{M}_{2 g-1}\right)=2 g-1-\left(f+f^{\prime}\right) . \operatorname{Now} \operatorname{dim}(U)=\operatorname{dim}(Z)$ and so

$$
\operatorname{dim}(U) \geq 3 g-3-\left(2 g-1-\left(f+f^{\prime}\right)\right)=g-2+f+f^{\prime} .
$$

The statement is also true for $\overline{\mathcal{R}}_{g}^{\left(f, f^{\prime}\right)}$ since $\mathcal{R}_{g}^{\left(f, f^{\prime}\right)}$ is open and dense in it.

Remark 5.3. The hypothesis in Proposition 5.2 that $\mathcal{R}_{g}^{\left(f, f^{\prime}\right)} \neq \emptyset$ is not superfluous. When $p=3$, then $\mathcal{R}_{2}^{(0,0)}=\emptyset[9$, Theorem 6.1].

Remark 5.4. The strategy of the proof of Proposition 5.2 does not give much information for covers of degree $\ell \geq 3$ because $g_{Y}$ is too big relative to $3 g-3$.

5.2. Increasing the $p$-rank of the Prym variety. We show that geometric information about $\mathcal{R}_{g}^{\left(f, f^{\prime}\right)}$ can be used to deduce geometric information about $\mathcal{R}_{g}^{\left(f, F^{\prime}\right)}$ when $f^{\prime} \leq F^{\prime} \leq g-1$.

Proposition 5.5. Let $g \geq 2$. If $\mathcal{R}_{g}^{\left(f, f^{\prime}\right)}$ is non-empty and has a component of dimension $g-2+f+f^{\prime}$ in characteristic $p$, then $\mathcal{R}_{g}^{\left(f, F^{\prime}\right)}$ is non-empty and has a component of dimension $g-2+f+F^{\prime}$ in characteristic $p$ for each $F^{\prime}$ such that $f^{\prime} \leq F^{\prime} \leq g-1$.

Proof. Let $S_{f^{\prime}}$ be a component of $\mathcal{R}_{g}^{\left(f, f^{\prime}\right)}$ having dimension $g-2+f+f^{\prime}$. Then $S_{f^{\prime}}$ is contained in $W_{g}^{f}:=\Pi^{-1}\left(\mathcal{M}_{g}^{f}\right)$. Each component of the latter has dimension $2 g-3+f$ since $\Pi: \mathcal{R}_{g} \rightarrow \mathcal{M}_{g}$ is finite and flat and $\mathcal{M}_{g}^{f}$ is pure of dimension $2 g-3+f$ by [9, Theorem 2.3]. Thus $S_{f^{\prime}}$ has codimension $g-1-f^{\prime}$ in $W_{g}^{f}$. Also, the generic geometric point of $W_{g}^{f}$ represents a cover $\pi$ such that the Prym $P_{\pi}$ has $p$-rank $g-1$ by Theorem 4.5 . 
Consider the forgetful morphism $\tau_{g}: \mathcal{R}_{g} \rightarrow \mathcal{M}_{2 g-1}$ which sends the point representing $\pi: Y \rightarrow X$ to the point representing $Y$. Since $g \geq 3$, the map $\tau_{g}$ is finite-to- 1 . Now $\tau_{g}\left(S_{f^{\prime}}\right) \subset \mathcal{M}_{2 g-1}^{f+f^{\prime}}$ and $\tau_{g}\left(W_{g}^{f}\right) \subset \mathcal{M}_{2 g-1}^{f+g-1}$. Thus the $p$-ranks and the dimensions for $\tau_{g}\left(S_{f^{\prime}}\right)$ and $\tau_{g}\left(W_{g}^{f}\right)$ both differ by exactly $g-1-f^{\prime}$.

By purity, the $p$-rank can only change in codimension 1. It follows that there is a nested sequence $T_{f^{\prime}} \subset \cdots \subset T_{i} \subset \cdots \subset T_{g-1}$, indexed by $i$ from $f^{\prime}$ to $g-1$, with $T_{f^{\prime}}=\tau_{g}\left(S_{0}\right)$ and $T_{g-1}=\tau_{g}\left(W_{g}^{f}\right)$, such that $\operatorname{dim}\left(T_{i}\right)=g-2+f+i$ and the generic geometric point of $T_{i}$ represents a curve $Y_{i}$ with $p$-rank $f+i$.

Then $T_{i}$ is in the image of $\tau_{g}$, so there is a sequence $S_{f^{\prime}} \subset \cdots \subset S_{i} \cdots \subset W_{g}^{f}$ such that $\tau_{g}\left(S_{i}\right)=T_{i}$. Thus $\operatorname{dim}\left(S_{i}\right)=g-2+f+i$. Also, the generic geometric point of $S_{i}$ represents an unramified double cover $\pi: Y_{i} \rightarrow X_{i}$ such that $Y_{i}$ has $p$-rank $f+i$ and $X_{i}$ has genus $g$ and $p$-rank $f$; it follows that $P_{\pi_{i}}$ has $p$-rank $i$. Thus $R_{g}^{\left(f, f^{\prime}\right)}$ contains an open dense subset of $S_{i}$, which we denote again by $S_{i}$ at the risk of causing confusion.

The next claim is that $S_{i}$ is open and dense in a component of $R_{g}^{(f, i)}$ for $f^{\prime} \leq i \leq g-1$. This is true for $i=f^{\prime}$ by hypothesis. If it is not true for all $i$, let $j$ be the minimal index for which it is false. Then $S_{j-1}$ has codimension at least 2 inside a component $\Sigma$ of $R_{g}^{(f, j)}$, and $\tau_{g}\left(S_{j-1}\right)$ has codimension at least 2 in $\tau_{g}(\Sigma)$. This contradicts purity, since the $p$-rank drops by 1 on a subset of codimension 2 . This completes the proof.

\section{Results FOR LOW GENUS WHEN $\ell=2$}

This section contains results about non-ordinary Pryms of unramified double covers of curves of low genus $g=2$ and $g=3$. Since this section is only about double covers, the subscript $\ell=2$ is dropped from the notation for simplicity.

Recall that $\mathcal{A}_{g}^{f}$ is irreducible for all $g \geq 2$ and $0 \leq f \leq g$ except $(g, f)=(2,0)$ [5. Theorem A]. When either $g=2, f=1,2$ or $g=3,0 \leq f \leq 3$, the image of $\mathcal{M}_{g}^{f}$ under the Torelli map is open and dense in $\mathcal{A}_{g}^{f}$ and thus $\mathcal{M}_{g}^{f}$ is irreducible as well.

6.1. Base Case: Genus 2. This section contains a proof that $\mathcal{R}_{2}^{\left(f, f^{\prime}\right)}$ is non-empty with the expected dimension for all six choices of $\left(f, f^{\prime}\right)$ when $p \geq 5$.

Proposition 6.1. Let $g=2,0 \leq f \leq 2$, and $0 \leq f^{\prime} \leq 1$. Then $\mathcal{R}_{2}^{\left(f, f^{\prime}\right)}$ is non-empty (except when $p=3, f=0,1$, and $\left.f^{\prime}=0\right)$ and each of its components has dimension $f+f^{\prime}$.

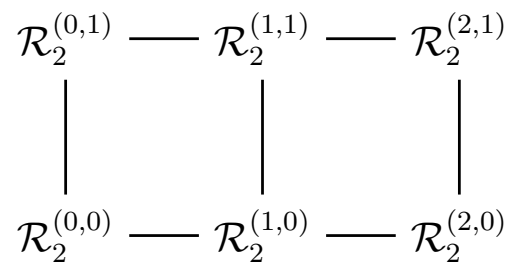

FiguRE 5. The $p$-rank stratification of $\mathcal{R}_{2}$

Proof. By Lemma 3.3. $W_{2}^{f}$ is non-empty with dimension $1+f$ for $0 \leq f \leq 2$. If $f=1,2$, then $\mathcal{M}_{2}^{f}$ is irreducible and so $W_{2}^{f}$ is irreducible by Proposition 4.3 .

By [9, Section 7.1], $\mathcal{R}_{2}^{(1,0)}$ and $\mathcal{R}_{2}^{(0,0)}$ are empty when $p=3$. 
- $(0,0)$. By [9, Theorem 6.1], if $p \geq 5$, then $\mathcal{R}_{2}^{(0,0)}$ is nonempty with dimension 0 .

- $(0,1)$. When $f=0$, then $W_{2}^{0}=\mathcal{R}_{2}^{(0,1)} \cup \mathcal{R}_{2}^{(0,0)}$. So $\mathcal{R}_{2}^{(0,1)}$ is open and dense in $W_{2}^{0}$ and thus $\operatorname{dim}\left(\mathcal{R}_{2}^{(0,1)}\right)=1$.

- $(2,1)$. Since $W_{2}^{2}$ is open and dense in $\mathcal{R}_{2}$, which contains $\mathcal{R}_{2}^{(0,1)}$, the generic point of $W_{2}^{2}$ has $f^{\prime}=1$. This implies that $\mathcal{R}_{2}^{(2,1)}$ is open and dense in $W_{2}^{2}$ and $\operatorname{dim}\left(\mathcal{R}_{2}^{(2,1)}\right)=3$.

- $(1,1)$. Purity, applied to $\mathcal{R}_{2}^{(0,1)} \subset \mathcal{R}_{2}$, shows that $\mathcal{R}_{2}^{(1,1)}$ is non-empty with dimension 2. Thus $\mathcal{R}_{2}^{(1,1)}$ is open and dense in $W_{2}^{1}$.

- $(2,0)$. The fiber product construction in Section 9.1 shows that $\operatorname{dim}\left(V_{2}^{0}\right)=2$. Namely, a point of $\mathcal{A}_{1}^{0}$ is represented by a supersingular elliptic curve $E_{\lambda}$. By (5) in Section 9.1. there is a 2-dimensional family of curves $X$ with an unramified double cover $\pi: Y \rightarrow X$ with $P_{\pi} \sim E_{\lambda}$.

Note that $\mathcal{R}_{2}^{(2,0)}$ is non-empty and open and dense in $V_{2}^{0}$ : if $p=3$, then $\mathcal{R}_{2}^{(2,0)}=V_{2}^{0}$ since $\mathcal{R}_{2}^{(0,0)}$ and $\mathcal{R}_{2}^{(1,0)}$ are empty; if $p \geq 5$, then $W_{2}^{1}$ is irreducible with dimension 2 and generic $f^{\prime}=1$ and so no component of $V_{2}^{0}$ is contained in $W_{2}^{1}$.

- $(1,0)$. If $p \geq 5$, applying purity to $\mathcal{R}_{2}^{(0,0)} \subset V_{2}^{0}$ shows that $\mathcal{R}_{2}^{(1,0)}$ is non-empty. Also $\mathcal{R}_{2}^{(1,0)} \subset W_{2}^{1}$ which is irreducible of dimension 2 and generic $f^{\prime}=1$. Thus $\operatorname{dim}\left(\mathcal{R}_{2}^{(1,0)}\right) \leq 1$. By Proposition 5.2 , every component of $\mathcal{R}_{2}^{(1,0)}$ has dimension 1 .

Remark 6.2. When $g=2$, then each of the 15 connected unramified $\mathbb{Z} / 2$-covers $\pi: Y \rightarrow X$ arises via a fiber product construction. For a fixed (small) prime $p$, it is thus computationally feasible to find equations for curves represented by points of $\mathcal{R}_{2}^{\left(f, f^{\prime}\right)}$. However, if $f+f^{\prime}$ is small, then it is not feasible to prove that $\mathcal{R}_{2}^{\left(f, f^{\prime}\right)}$ is non-empty for all primes $p$ using a computational perspective; this is explained in more detail in Section 9.1.

Remark 6.3. Consider $\mathcal{M}_{1 ; 2}^{0}$, whose points represent supersingular elliptic curves with 2 marked points. Then $\kappa_{0, I I}\left(\mathcal{M}_{1 ; 2}^{0}\right)$ has dimension 1 and is fully contained in $\partial \mathcal{R}_{2}^{(1,0)}$.

6.2. Base case: $g=3$.

Proposition 6.4. Let $\ell=2$ and $p \geq 3$. Let $g=3$ and $0 \leq f \leq 3$ (with $f \neq 0,1$ when $p=3$ ). Then $\Pi^{-1}\left(\mathcal{M}_{3}^{f}\right)$ is irreducible and $\mathcal{R}_{3}^{(f, 1)}=\Pi^{-1}\left(\mathcal{M}_{3}^{f}\right) \cap V_{3}^{1}$ is non-empty with dimension $2+f$.

Proof. For $0 \leq f \leq 3, \mathcal{M}_{3}^{f}$ is irreducible and so $\Pi^{-1}\left(\mathcal{M}_{3}^{f}\right)$ is irreducible by Proposition 4.3. Also $\operatorname{dim}\left(\Pi^{-1}\left(\mathcal{M}_{3}^{f}\right)\right)=3+f$. By Theorem 4.5, the Prym of the unramified $\mathbb{Z} / 2$-cover represented by the generic point of $\Pi^{-1}\left(\mathcal{M}_{3}^{f}\right)$ has $p$-rank $f^{\prime}=2$. By Proposition 5.2, if $\mathcal{R}_{3}^{(f, 1)}=\Pi^{-1}\left(\mathcal{M}_{3}^{f}\right) \cap V_{3}^{1}$ is non-empty, then its components have dimension $2+f$.

Recall that $\mathcal{R}_{2}^{(f, 0)}$ is non-empty and has dimension $f$ by Proposition 6.1 when $f=1,2$ and by [9, Theorem 6.1] (except when $p=3$ and $f=0,1$ ).

If $0 \leq f \leq 2$, consider $I=\kappa_{2: 1}\left(\mathcal{R}_{2 ; 1}^{(f, 0)} \times \mathcal{R}_{1 ; 1}^{(0,0)}\right) \subset \overline{\mathcal{R}}_{3}$. Then $I$ is non-empty. The choice of base point increases the dimension by 1 so $\operatorname{dim}(I)=\operatorname{dim}\left(\overline{\mathcal{R}}_{2}^{(f, 0)}\right)+1=f+1$. If $f=3$, consider $I=\kappa_{2: 1}\left(\mathcal{R}_{2 ; 1}^{(2,0)} \times \mathcal{R}_{1 ; 1}^{(1,0)}\right) \subset \overline{\mathcal{R}}_{3}$. Then $I \neq \emptyset$ and $\operatorname{dim}(I)=4$.

By Lemmas 3.6 and 3.7 , $I$ is contained in a component $T$ of $\overline{\mathcal{R}}_{3}^{(f, 1)}$. Now $\operatorname{dim}(T) \geq f+2$ by Proposition 5.2. The generic point of $T$ is not contained in $\Delta_{2: 1}\left[\overline{\mathcal{R}}_{3}^{(f, 1)}\right]$ since the dimension 
of the latter is bounded by $f+1$. Moreover, from the construction of $I$, the generic point of $T$ is not contained in any other boundary component and is thus in $\mathcal{R}_{3}^{(f, 1)}$.

\section{Non-ORdinary PRYMS of UnRAMified DOUble COVERS}

In this section, we demonstrate the existence of smooth curves of given genus and $p$-rank having an unramified double cover whose Prym is not ordinary. Since this section is only about double covers, the subscript $\ell=2$ is dropped from the notation for simplicity.

7.1. Almost ordinary. Theorem 1.2(1) follows from Theorem 7.1, which states that there is a codimension one condition on a generic curve $X$ of genus $g$ and $p$-rank $f$ for which the Prym of an unramified double cover $\pi: Y \rightarrow X$ is almost ordinary. The almost ordinary condition means that the $p$-rank of $P_{\pi}$ is $g-2=\operatorname{dim}\left(P_{\pi}\right)-1$.

Consider the stratum $\mathcal{R}_{g}^{(f, g-2)}=W_{g}^{f} \cap V_{g}^{g-2}$ whose points represent unramified double covers $\pi: Y \rightarrow X$ such that $X$ is a smooth curve of genus $g$ and $p$-rank $f$ and such that $P_{\pi}$ has $p$-rank $f^{\prime}=g-2$ (or, equivalently, such that $P_{\pi}$ is almost ordinary).

Theorem 7.1. Let $\ell=2$ and $p \geq 3$. Let $g \geq 2$ and $0 \leq f \leq g$ (with $f \geq 2$ when $p=3$ ). Let $f^{\prime}=g-2$. Then $\mathcal{R}_{g}^{(f, g-2)}$ is non-empty and each of its components has dimension $2 g-4+f$.

More generally, let $S$ be a component of $\mathcal{M}_{g}^{f}$. Then the locus of points of $\Pi^{-1}(S)$ representing unramified double covers for which the Prym $P_{\pi}$ is almost ordinary is non-empty and codimension 1 in $\Pi^{-1}(S)$ (dimension $2 g-4+f$ ).

Proof. The first statement follows from the second since every component of $\mathcal{R}_{g}^{(f, g-2)}$ is contained in $\Pi^{-1}(S)$ for some component $S$ of $\mathcal{M}_{g}^{f}$. It thus suffices to prove that $\Pi^{-1}(S) \cap$ $V_{g}^{g-2}$ is non-empty and its components have dimension $2 g-4+f$.

Dimension: Let $T$ be a component of $\Pi^{-1}(S) \cap V_{g}^{g-2}$. Then Proposition 5.2 implies that $\operatorname{dim}(T) \geq 2 g-4+f$. The generic point of $\Pi^{-1}(S)$ represents a cover whose Prym has $p$-rank $f^{\prime}=g-1$ by Theorem 4.5 (or Proposition 6.1 if $g=2$ and $f=0$ ). Thus $\operatorname{dim}(T)=\operatorname{dim}\left(\Pi^{-1}(S)\right)-1=2 g-4+f$. It thus suffices to show $\Pi^{-1}(S) \cap V_{g}^{g-2}$ is nonempty.

Base cases: When $g=2$ and $0 \leq f \leq 2$, then $\Pi^{-1}(S) \cap V_{2}^{0}$ is non-empty with dimension $f$ by Proposition 6.1 (unless $p=3$ and $f=0,1)$. When $g=3$ and $0 \leq f \leq 3$, then $\Pi^{-1}\left(\mathcal{M}_{3}^{f}\right)$ is irreducible and $\Pi^{-1}\left(\mathcal{M}_{3}^{f}\right) \cap V_{g}^{1}$ is non-empty with dimension $2+f$ by Proposition 6.4.

Strategy: Suppose $g \geq 4$. Let $\bar{S}$ be the closure of $S$ in $\overline{\mathcal{M}}_{g}^{f}$. The plan is to show that $\Pi^{-1}(\bar{S}) \cap \bar{V}_{g}^{g-2}$ is non-empty and that one of its components is not contained in $\partial \mathcal{R}_{g}$.

Non-empty: Let $g_{1}=3$ and $g_{2}=g-3$. Choose $f_{1}, f_{2}$ such that $f_{1}+f_{2}=f$ with $0 \leq f_{i} \leq g_{i}$. By Proposition 4.1, there are components $S_{i ; 1}$ of $\overline{\mathcal{M}}_{g_{i} ; 1}^{f_{i}}$ such that

$$
\kappa_{g_{1}: g_{2}}\left(S_{1 ; 1} \times S_{2 ; 1}\right) \subset \bar{S} .
$$

Recall the forgetful map $\psi_{M}: \mathcal{M}_{g ; 1} \rightarrow \mathcal{M}_{g}$ from Section 2.3. Let $S_{i}=\psi_{M}\left(S_{i ; 1}\right)$ which is an irreducible component of $\overline{\mathcal{M}}_{g_{i}}^{f_{i}}$.

The Prym of the cover represented by the generic point of $\Pi^{-1}\left(S_{2}\right)$ has $p$-rank $f_{2}^{\prime}=g_{2}-1$ by Theorem 4.5. By Proposition 4.3. $\Pi^{-1}\left(S_{1}\right)$ is irreducible. By Proposition 6.4, there exists 
a point of $\Pi^{-1}\left(S_{1}\right)$ representing a cover whose Prym has $p$-rank $f_{1}^{\prime}=g_{1}-2=1$. Since $\overline{\mathcal{M}}_{3}^{f_{1}}$ is irreducible, $\Pi^{-1}\left(S_{1}\right)=\bar{W}_{3}^{f_{1}}$. Consider

$$
N:=\kappa_{g_{1}: g_{2}}\left(\Pi^{-1}\left(S_{1 ; 1}\right) \times \Pi^{-1}\left(S_{2 ; 1}\right)\right) \subset \Pi^{-1}(\bar{S}) .
$$

By Lemma 3.7, $N$ contains a point representing a cover whose Prym has $p$-rank $f^{\prime}=f_{1}^{\prime}+$ $f_{2}^{\prime}+1=g-2$, i.e., whose Prym is almost ordinary. Thus $\Pi^{-1}(\bar{S}) \cap V_{g}^{g-2}$ is non-empty.

Generically smooth: Let $T$ be a component of $\Pi^{-1}(\bar{S}) \cap \bar{V}_{g}^{g-2}$ containing $N$. By the remarks above, $T$ intersects the image of

$$
\kappa_{g_{1}: g_{2}}: \overline{\mathcal{R}}_{g_{1} ; 1}^{\left(f_{1}, 1\right)} \times \bar{W}_{g_{2} ; 1}^{f_{2}} \rightarrow \Delta_{3: g-3}\left[\overline{\mathcal{R}}_{g}^{(f, g-2)}\right] .
$$

This image has dimension

$$
\left(2+f_{1}\right)+1+\left(2(g-3)-3+f_{2}\right)+1=2 g-5+f .
$$

By Proposition 5.2, $\operatorname{dim}(T) \geq 2 g-4+f$. Thus the generic point $\tau$ of $T$ is not contained in $\Delta_{3: g-3}\left[\overline{\mathcal{R}}_{g}\right]$. Furthermore, $\tau$ is not contained in any other component of $\partial \mathcal{R}_{g}$ because the generic points of $S_{1}$ and $S_{2}$ represent smooth curves. Thus $\tau \subset \Pi^{-1}(S) \cap V_{g}^{g-2}$.

7.2. Pryms with low $p$-rank. As an application, we demonstrate the existence of smooth curves of given genus and $p$-rank having an unramified double cover whose Prym has any $p$-rank between $\frac{g}{2}-1$ and $g-3$.

Theorem 7.2. Let $p \geq 5$. Let $g \geq 2$ and write $g=3 r+2 s$ for integers $r, s \geq 0$. Let $0 \leq f \leq g$. Let $2 r+s-1 \leq f^{\prime} \leq g-1$. Then $\mathcal{R}_{g}^{\left(f, f^{\prime}\right)}$ is non-empty and has a component of dimension $g-2+f+f^{\prime}$ in characteristic $p$.

Proof. In light of Proposition 5.5, it suffices to prove the result when $f^{\prime}=2 r+s-1$. The proof is by induction on $r+s$. In the base case $(r, s)=(0,1)$, then $g=2$ and the result is true by Proposition 6.1. In the base case $(r, s)=(1,0)$, then $g=3$ and the result is true by Proposition 6.4. As an inductive hypothesis, suppose that the result is true for all pairs $\left(r^{\prime}, s^{\prime}\right)$ such that $1 \leq r^{\prime}+s^{\prime}<r+s$.

Case 1: suppose $r \geq 1$. Let $g_{1}=3$ and $g_{2}=g-3$. There exist $f_{1}, f_{2}$ such that $f_{1}+f_{2}=f$ and $0 \leq f_{1} \leq g_{1}$ and $0 \leq f_{2} \leq g_{2}$. Let $f_{1}^{\prime}=1$ and $f_{2}^{\prime}=2 r+s-3$. By Proposition 6.4, $\mathcal{R}_{3}^{\left(f_{1}, 1\right)}$ is non-empty and has a component $S_{1}$ of dimension $d_{1}=2+f_{1}$. (The points of $S_{1}$ represent unramified double covers $\pi_{1}: Y_{1} \rightarrow X_{1}$ of a smooth curve of genus $g_{1}$ and $p$-rank $f_{1}$, such that $P_{\pi_{1}}$ has $p$-rank 1.)

By the inductive hypothesis applied to $(r-1, s)$, it follows that $\mathcal{R}_{g_{2}}^{\left(f_{2}, 2 r+s-3\right)}$ is non-empty and has a component $S_{2}$ of dimension $d_{2}=g_{2}-2+f_{2}+f_{2}^{\prime}$. (The points of $S_{2}$ represent unramified double covers $\pi_{2}: Y_{2} \rightarrow X_{2}$ of a smooth curve of genus $g_{2}$ and $p$-rank $f_{2}$, such that $P_{\pi_{2}}$ has $p$-rank $f_{2}^{\prime}$.) Adding a marking increases the dimension by 1 , so $U_{1}:=\psi_{R}^{*}\left(S_{1}\right)=$ $S_{1} \times_{\mathcal{R}_{3}} \mathcal{R}_{3 ; 1}$ has dimension $d_{1}+1=3+f_{1}$ and $U_{2}:=\psi_{R}^{*}\left(S_{2}\right)=S_{2} \times_{\mathcal{R}_{g_{2}}} \mathcal{R}_{g_{2} ; 1}$ has dimension $d_{2}+1=g_{2}-1+f_{2}+f_{2}^{\prime}$.

Let $\mathcal{K}$ be a component of $\kappa_{3, g_{2}}\left(U_{1} \times U_{2}\right)$; then $\mathcal{K}$ has dimension $d_{1}+d_{2}+2$. By Lemmas 3.6 and 3.7. $\mathcal{K}$ is contained in a component $\mathcal{Z}$ of $\overline{\mathcal{R}}_{g_{1}+g_{2}}^{\left(f_{1}+f_{2} f_{1}^{\prime}+f_{2}^{\prime}+1\right)}=\overline{\mathcal{R}}_{g}^{(f, 2 r+s-1)}$. In other words, the points of $\mathcal{K}$ represent unramified double covers of curves (of compact type) having genus 
$g$ and $p$-rank $f$ whose Prym varieties have $p$-rank $f^{\prime}$. By Lemma 3.1, the dimension of $\mathcal{Z}$ is at most

$$
d_{1}+d_{2}+3=\left(g_{2}+3\right)-2+\left(f_{1}+f_{2}\right)+(2 r+s-1)=g-2+f+f^{\prime} .
$$

Also $\operatorname{dim}(\mathcal{Z}) \geq g-2+f+f^{\prime}$ by purity. Thus $\operatorname{dim}(\mathcal{Z})=g-2+f+f^{\prime}$ and the generic point of $\mathcal{Z}$ is not contained in $\mathcal{K}$. The generic geometric points of $S_{1}$ and $S_{2}$ represent unramified double covers of smooth curves by hypothesis. Thus the generic geometric point of $\mathcal{Z}$ is not contained in any other boundary component of $\overline{\mathcal{R}}_{g}$ and so it represents an unramified double cover of a smooth curve.

Case 2: suppose $s \geq 1$. Let $g_{1}=2$ and $g_{2}=g-2$. There exist $f_{1}, f_{2}$ such that $f_{1}+f_{2}=f$ and $0 \leq f_{1} \leq g_{1}$ and $0 \leq f_{2} \leq g_{2}$. Let $f_{1}^{\prime}=0$ and $f_{2}^{\prime}=2 r+s-2$. By Proposition 6.1, $\mathcal{R}_{2}^{\left(f_{1}, 0\right)}$ is non-empty and has a component $S_{1}$ of dimension $d_{1}=f_{1}$. By the inductive hypothesis applied to $(r, s-1)$, it follows that $\mathcal{R}_{g_{2}}^{\left(f_{2}, 2 r+s-2\right)}$ is non-empty and has a component $S_{2}$ of dimension $d_{2}=g_{2}-2+f_{2}+(2 r+s-2)$. The rest of the proof follows the same reasoning as in Case (1).

Corollary 7.3. Let $\ell=2$ and $p \geq 5$. Let $g \geq 4$ and $0 \leq f \leq g$. Suppose $\frac{g}{2}-1 \leq f^{\prime} \leq g-3$. Then $\mathcal{R}_{g}^{\left(f, f^{\prime}\right)}$ is non-empty and has a component of dimension $g-2+f+f^{\prime}$. In particular, there exists a smooth curve $X / \overline{\mathbb{F}}_{p}$ of genus $g$ and $p$-rank $f$ having an unramified double cover $\pi: Y \rightarrow X$ for which the Prym $P_{\pi}$ has $p$-rank $f^{\prime}$.

Proof. If $g$ is even, let $r=0$ and $s=g / 2$. If $g$ is odd, let $r=1$ and $s=(g-3) / 2$. In either case, the condition $\frac{g}{2}-1 \leq f^{\prime} \leq g-3$ implies that the hypothesis $2 r+s-1 \leq f^{\prime} \leq g-1$ in Theorem 7.2 is satisfied and the result follows from Theorem 7.2 .

\section{Applichtions to Theta Divisors}

8.1. Background. Recall the definition of the theta divisor from [19, Section 1.1]. Given a relative curve $X / S$, let $X^{1}$ be the curve induced by base change by the absolute Frobenius of $S$. Consider the relative Frobenius morphism $F: X \rightarrow X^{1}$. The sheaf of locally exact differentials $B$ is the image of $F_{*} d: F_{*}\left(\mathcal{O}_{X}\right) \rightarrow F_{*}\left(\Omega_{X}^{1}\right)$. There is an exact sequence of $\mathcal{O}_{X^{1}}$-modules:

$$
0 \rightarrow \mathcal{O}_{X^{1}} \rightarrow F_{*}\left(\mathcal{O}_{X}\right) \stackrel{F_{*} d}{\rightarrow} B \rightarrow 0 .
$$

Also, $B$ is the kernel of the Cartier operator $C: F_{*}\left(\Omega_{X}^{1}\right) \rightarrow \Omega_{X^{1}}^{1}$, and there is an exact sequence of $\mathcal{O}_{X^{1}}$-modules:

$$
0 \rightarrow B \rightarrow F_{*}\left(\Omega_{X}^{1}\right) \stackrel{C}{\rightarrow} \Omega_{X^{1}}^{1} \rightarrow 0 .
$$

Now $B$ is a vector bundle on $X^{1}$ of rank $p-1$ and slope $g-1$, where the slope is the quotient of the degree by the rank. More precisely, if $X^{1}$ is not smooth, then $B$ is a torsion-free sheaf, which is locally free of rank $p-1$ outside the singularities of $X^{1}$.

By [17, Theorem 4.1.1], $B$ admits a theta divisor $\Theta_{X}$. This is a positive Cartier divisor on the Jacobian $J^{1}$ of $X^{1}$ (the determinant of the universal cohomology). A point $a \in J^{1}(k)$ is in the support of $\Theta_{X}$ if and only if $H^{0}\left(X^{1}, B \otimes L_{a}\right) \neq 0$ where $L_{a} \in \operatorname{Pic}^{0}\left(X^{1}\right)$ is the invertible sheaf identified with $a$. 
8.2. The theta divisor. By work of Raynaud, the theta divisor $\theta_{X}$ determines whether unramified covers of the curve $X$ are ordinary. By [18, Proposition 1], $X$ is ordinary if and only if $\theta_{X}$ does not contain the identity of $J^{1}$.

To generalize this, consider a non-trivial point $a \in J^{1}[\ell]$ with $p \nmid \ell$. The point $a$ determines an unramified $\mathbb{Z} / \ell$-cover $\pi_{a}: Y_{a} \rightarrow X$, and an invertible sheaf $L_{a} \in \operatorname{Pic}^{0}\left(X^{1}\right)$ of order $\ell$. Denote the orbit of $a$ under $(\mathbb{Z} / \ell)^{*}$ as $\operatorname{Sat}(a)=\{i a \mid \operatorname{gcd}(i, \ell)=1\}$.

Proposition 8.1. [19, Proposition 2.1.4] Let $a \in J^{1}[\ell]$ be non-trivial with $p \nmid \ell$. The new part of $\pi_{a}: Y_{a} \rightarrow X$ is ordinary if and only if $\operatorname{Sat}(a)$ does not intersect the theta divisor $\Theta_{X}$.

Using the geometry of $\Theta_{X}$, Raynaud and Pop/Saidi prove:

Theorem 8.2. Let $X$ be a smooth projective $k$-curve of genus $g \geq 2$.

(1) [17, Theorem 4.3.1] For sufficiently large $\ell$, there is an unramified $\mathbb{Z} / \ell$-cover $\pi: Y \rightarrow$ $X$ such that $P_{\pi}$ is ordinary. It suffices to take $\ell>(p-1) 3^{g-1} g$ ! by [20, Remark 3.1.1].

(2) [18, Theorem 2] There is an unramified Galois cover $Z \rightarrow X$, with solvable prime-to-p Galois group, with a non-ordinary representation (so $Z$ is not ordinary).

(3) [16, Proposition 2.3] If $X$ is non-ordinary or if $J_{X}$ is simple then there is an unramified $\mathbb{Z} / \ell$-cover $\pi_{\ell}: Y_{\ell} \rightarrow X$ such that $P_{\pi_{\ell}}$ is not ordinary for infinitely many primes $\ell$.

8.3. Comparison with previous work. The results in this paper strengthen the results in Theorem 8.2 for a generic curve $X$ of genus $g$ and $p$-rank $f$ for all $g \geq 2$ and $0 \leq f \leq g$. Specifically, Theorem 1.1 removes the condition on $\ell$ in Theorem 8.2(1) and shows that all (not just one) of the Pryms of the $\mathbb{Z} / \ell$-covers of $X$ are ordinary, for a generic curve $X$ of genus $g$ and $p$-rank $f$. Theorem 1.2 and Corollary 7.3 are about double covers, rather than covers of unknown degree, and they determine the value of the $p$-rank of the Prym which gives more information than saying that the new part of the Prym is not ordinary.

8.4. New results on theta divisors. We apply Proposition 8.1 in the opposite direction from Raynaud and Pop/Saidi to complete the proofs of Theorems 1.1(2) and 1.2(2).

Theorem 8.3. Let $\ell \neq p$ be prime. Let $g \geq 2$ and $0 \leq f \leq g$ with $f \neq 0$ if $g=2$. Let $S$ be an irreducible component of $\mathcal{M}_{g}^{f}$. If $X$ is the curve represented by the generic point of $S$, then the theta divisor $\Theta_{X}$ of the Jacobian of $X$ does not contain any point of order $\ell$.

Proof. By Proposition 8.1, this statement is equivalent to Theorem 1.1(1) since the Prym is the new part of $\pi: Y \rightarrow X$.

Theorem 8.4. Let $\ell=2$. Let $g \geq 2$ and $0 \leq f \leq g$ (with $f \geq 2$ when $p=3$ ). Let $S$ be an irreducible component of $\mathcal{M}_{g}^{f}$. The locus of points of $S$ representing curves $X$ for which $\Theta_{X}$ contains a point of order 2 is non-empty with codimension 1 in $S$.

Proof. This follows from Theorem 1.2(1) by Proposition 8.1.

Remark 8.5. Let $g$ be odd and $d=(g-1) / 2$. If $X$ has genus $g$, then $J_{X}$ contains the top difference variety $V_{d}=X_{d}-X_{d}$, which consists of divisors of the form $\sum_{i=1}^{d} P_{i}-\sum_{i=1}^{d} Q_{i}$. By [6, Corollary 0.4], $V_{d}$ contains no points of order $\ell$ when $X$ is generic.

\section{ExAMPLES AND OPEN QUESTIONS}

This section contains examples for $g=2$, a question about Pryms of hyperelliptic curves, and questions about non-ordinary Pryms whose answers would generalize Theorem 1.2(1). 
9.1. The fiber product construction when $g=2$. We explain why the fiber product construction of unramified double covers is not useful for proving Proposition 6.1.

Suppose $X$ is a genus 2 curve and $f_{1}: X \rightarrow \mathbb{P}^{1}$ is a hyperelliptic cover branched above a set $B_{X}$ of cardinality 6 . For a set $B_{E} \subset B_{X}$ of cardinality 4 , let $f_{2}: E \rightarrow \mathbb{P}^{1}$ be the hyperelliptic cover branched above $B_{E}$. The fiber product $f: Y \rightarrow \mathbb{P}^{1}$ of $f_{1}$ and $f_{2}$ is a Klein four cover of $\mathbb{P}^{1}$. By Abhyankar's Lemma, the degree two subcover $\pi: Y \rightarrow X$ is unramified since $B_{E} \subset B_{X}$. Then $J_{Y} \sim J_{X} \oplus E$ by [11, Theorem B]. Thus $P_{\pi} \sim E$.

Furthermore, each of the 15 connected unramified double covers $\pi: Y \rightarrow X$ arises via the fiber product construction (from one of the 15 choices of $B_{E} \subset B_{X}$ ). This is because the hyperelliptic involution $\iota$ on $X$ fixes each point of order 2 on $J_{X}$ and thus extends to $Y$.

For $\lambda \in k-\{0,1\}$, let $E_{\lambda}: y_{2}^{2}=x(x-1)(x-\lambda)$. For distinct $t_{1}, t_{2} \in k-\{0,1, \lambda\}$, consider the genus two curve

$$
X: y_{1}^{2}=f_{\lambda}\left(t_{1}, t_{2}\right):=x(x-1)(x-\lambda)\left(x-t_{1}\right)\left(x-t_{2}\right) .
$$

As above, $E_{\lambda} \sim P_{\pi}$ for an unramified double cover $\pi: Y \rightarrow X$. One says that $\lambda$ is supersingular when $E_{\lambda}$ is supersingular.

Let $M_{\lambda}\left(t_{1}, t_{2}\right)$ be the matrix of the Cartier operator on $H^{0}\left(X, \Omega^{1}\right)$ with respect to the basis $\{d x / y, x d x / y\}$. Let $c_{i}$ be the coefficient of $x^{i}$ in $f_{\lambda}\left(t_{1}, t_{2}\right)^{(p-1) / 2}$. By [23, page 381],

$$
M_{\lambda}\left(t_{1}, t_{2}\right)=\left(\begin{array}{cc}
c_{p-1} & c_{p-2} \\
c_{2 p-1} & c_{2 p-2}
\end{array}\right) .
$$

Let $D_{\lambda}=\operatorname{det}\left(M_{\lambda}\left(t_{1}, t_{2}\right)\right)$ and let $S_{\lambda} \subset \mathbb{A}^{2}$ be the vanishing locus of $D_{\lambda}$. By [23, Theorem 2.2], $X$ is ordinary if and only if $D_{\lambda} \neq 0$; the $p$-rank of $X$ is the rank of $N_{\lambda}\left(t_{1}, t_{2}\right)=$ $M_{\lambda}\left(t_{1}, t_{2}\right)^{(p)} M_{\lambda}\left(t_{1}, t_{2}\right)$ (where $(p)$ means to raise each entry of the matrix to the $p$ th power).

(1) The case $\left(f, f^{\prime}\right)=(2,0)$. For each supersingular $\lambda$, to show $X$ is generically ordinary, one needs to check that $D_{\lambda} \in k\left[t_{1}, t_{2}\right]$ is non-zero.

(2) The case $\left(f, f^{\prime}\right)=(\leq 1,0)$. To show $\mathcal{R}_{2}^{(1,0)} \cup \mathcal{R}_{2}^{(0,0)} \neq \emptyset$, one needs to find $\lambda$ supersingular such that $D_{\lambda} \in k\left[t_{1}, t_{2}\right]$ is non-constant and $S_{\lambda}$ is not contained in the union $L$ of the lines $t_{i}=0, t_{i}=1, t_{i}=\lambda$, and $t_{1}=t_{2}$.

(3) The case $\left(f, f^{\prime}\right)=(0,0)$ for $p \geq 5$. To show $\operatorname{dim}\left(\mathcal{R}_{2}^{(0,0)}\right)=0$, one needs to show that $N_{\lambda}\left(t_{1}, t_{2}\right)$ has rank 1 (not 0 ) for every supersingular $\lambda$ and for each generic point of $S_{\lambda}$ not in $L$. To show $\mathcal{R}_{2}^{(0,0)} \neq \emptyset$, one needs to find $\lambda$ supersingular and distinct $t_{1}, t_{2} \in k-\{0,1, \lambda\}$ such that $N_{\lambda}\left(t_{1}, t_{2}\right)$ has rank 0 .

Example 9.1. (Example of Proposition 6.1) Let $p=5$. Let $\lambda=a^{4}$ for a root $a$ of $x^{2}+4 x+2$. Then $E_{\lambda}$ is supersingular and

$$
D_{\lambda}=\left(t_{1}+4 t_{2}\right)^{2}\left(t_{1}^{2} t_{2}+t_{1} t_{2}^{2}+a^{17} t_{1}^{2}+a^{17} t_{2}^{2}+a^{5} t_{1} t_{2}+a^{4} t_{1}+a^{4} t_{2}\right) .
$$

(1) $\left(f, f^{\prime}\right)=(2,0)$. Since $D_{\lambda} \neq 0, X$ is generically ordinary.

(2) $\left(f, f^{\prime}\right)=(1,0)$. When $\left(t_{1}, t_{2}\right)=\left(a^{16}, a\right)$, then $f_{X}=1$.

(3) $\left(f, f^{\prime}\right)=(0,0)$. By [9, Section 7.2], there is exactly one unramified double cover $\pi: Y \rightarrow X$ up to isomorphism such that $X$ has genus 2 and $Y$ has $p$-rank 0 . An equation for $X$ is $y^{2}=x\left(x^{4}+x^{3}+2 x+3\right)$.

9.2. The hyperelliptic case. We expect there is an analogue of Theorem 4.5 for the hyperelliptic locus $\mathcal{H}_{g}$. One can ask if the Prym of the cover represented by the generic point of each irreducible component of $\Pi_{\ell}^{-1}\left(\mathcal{H}_{g}^{f}\right)$ is ordinary for $1 \leq f \leq g$. Propositions 4.1 and 
4.4 are true (for $i=1$ ) for $\mathcal{H}_{g}^{f}\left[2\right.$, Corollary 3.13] and Theorem 4.2 is true for $\mathcal{H}_{g}^{f}$ when $f>0$ (or for $f=0$ and $\ell>>0$ ) [2, Theorems 5.2, 5.7]. However, there may be complications with Propositions 3.4. 3.5 for $\mathcal{H}_{g}^{f}$, especially when $\ell=2$.

\subsection{A question for $g=3$ about Pryms of $p$-rank 0 .}

Question 9.2. For a prime $p$, is $\mathcal{R}_{3}^{(0,0)}$ non-empty? Does there exist an unramified double cover $\pi: Y \rightarrow X$ of a smooth curve $X / \overline{\mathbb{F}}_{p}$ of genus 3 such that $Y$ has $p$-rank 0 ?

The answer to Question 9.2 is yes when $p=3$ by [9, Example 5.5] but is unknown for $p \geq 5$. By [9, Proposition 4.2], if it is non-empty, then $\operatorname{dim}\left(\mathcal{R}_{3}^{(0,0)}\right)=1$; however, there are components of $\partial \bar{R}_{3}^{(0,0)}$ which have dimension 1 or 2 .

9.4. Non-ordinary Pryms for odd degree cyclic covers. It is unknown whether Theorem 7.1 can be generalized to the case $\ell \geq 3$, for a given prime $p$.

Question 9.3. Suppose $\ell \neq p$ is an odd prime. For which $(g, f)$ does there exist a curve $X$ of genus $g$ and $p$-rank $f$ with an unramified $\mathbb{Z} / \ell$-cover $\pi: Y \rightarrow X$ such that $P_{\pi}$ is non-ordinary?

Example 9.4. [15, Section 6] Let $p=2$ and $g=2$ and $\ell=3$. If $X$ is a curve of genus 2 which is not ordinary $(f<2)$, then the Prym of every unramified $\mathbb{Z} / 3$-cover of $X$ is ordinary.

9.5. A question about purity. Let $\ell=2$ and $p \geq 3$. The points of $\mathcal{R}_{g}^{\left(f, f^{\prime}\right)}=W_{g}^{f} \cap V_{g}^{f^{\prime}}$ represent unramified double covers $\pi: Y \rightarrow X$ such that $X$ is a smooth curve of genus $g$ and $p$-rank $f$ and $P_{\pi}$ has $p$-rank $f^{\prime}$. By Proposition 5.2 , $\operatorname{dim}\left(\mathcal{R}_{g ; 2}^{\left(f, f^{\prime}\right)}\right) \geq g-2+f+f^{\prime}$.

Question 9.5. Let $g \geq 2$ and $0 \leq f \leq g$ and $0 \leq f^{\prime} \leq g-1$. If $\mathcal{R}_{g ; 2}^{\left(f, f^{\prime}\right)}$ is non-empty, do all its components have dimension exactly $g-2+f+f^{\prime}$ ?

The answer to Question 9.5 is yes for any $0 \leq f \leq g$ when:

(1) $f^{\prime}=g-1$ by Theorem 4.5 (or Proposition 6.1);

(2) and $f^{\prime}=g-2$ (with $f \geq 2$ when $p=3$ ) by Theorem 7.1 .

One complication in answering Question 9.5 for $f^{\prime}<g-2$ is that there are families of singular curves in $\overline{\mathcal{R}}_{g ; 2}^{\left(f, f^{\prime}\right)}$ whose dimension exceeds $g-2+f+f^{\prime}$ as in Remark 6.3.

9.6. Pryms with $p$-rank zero. Let $\ell=2$ and $p \geq 5$ and $g=3$. Consider $\bar{V}_{3}^{1}=\operatorname{Pr}_{3}^{-1}\left(\tilde{\mathcal{A}}_{2}^{1}\right)$ whose points represent unramified double covers $\pi: Y \rightarrow X$, where $X$ has genus 3 and $P_{\pi}$ has $p$-rank 1. By [21, Theorem 4.2, equations 3.14-3.16], $V_{3}^{1}$ has one component of dimension 5 , and three exceptional components of lower dimension.

In $\bar{V}_{3}^{1}$ is the locus $V_{3}^{0}$ (additional constraint that $f^{\prime}=0$ ). By [21, Theorem 4.2], $\operatorname{dim}\left(V_{3}^{0}\right)=$ $3+\operatorname{dim}\left(\mathcal{A}_{2}^{0}\right)=4$. If $p>11$, then $\mathcal{A}_{2}^{0}$ is not irreducible by [12, Theorem 5.8], and so $V_{3}^{0}$ is not irreducible. Also in $\bar{V}_{3}^{1}$ is the locus $W_{3}^{2} \cap \bar{V}_{3}^{1}$, (additional constraint that $f=2$ ). By Proposition 6.4. $\operatorname{dim}\left(W_{3}^{2} \cap \bar{V}_{3}^{1}\right)=4$; it is not known whether it is irreducible.

Question 9.6. Is $\operatorname{dim}\left(W_{3}^{2} \cap V_{3}^{0}\right)=3$ ?

\section{REFERENCES}

[1] Jeffrey D. Achter and Rachel Pries. Monodromy of the p-rank strata of the moduli space of curves. Int. Math. Res. Not. IMRN, (15):Art. ID rnn053, 25, 2008.

[2] Jeffrey D. Achter and Rachel Pries. The $p$-rank strata of the moduli space of hyperelliptic curves. Adv. Math., 227(5):1846-1872, 2011. 
[3] Siegfried Bosch, Werner Lütkebohmert, and Michel Raynaud. Néron models, volume 21 of Ergebnisse der Mathematik und ihrer Grenzgebiete (3) [Results in Mathematics and Related Areas (3)]. Springer-Verlag, Berlin, 1990.

[4] Irene I. Bouw. The p-rank of ramified covers of curves. Compositio Math., 126(3):295-322, 2001.

[5] Ching-Li Chai and Frans Oort. Monodromy and irreducibility of leaves. Ann. of Math. (2), 173(3):13591396, 2011.

[6] Alessandro Chiodo, David Eisenbud, Gavril Farkas, and Frank-Olaf Schreyer. Syzygies of torsion bundles and the geometry of the level $\ell$ modular variety over $\overline{\mathcal{M}}_{g}$. Invent. Math., 194(1):73-118, 2013.

[7] Alessandro Chiodo and Gavril Farkas. Singularities of the moduli space of level curves. To appear in the Journal of the European Mathematical Society, http://arxiv.org/pdf/1205.0201v3.pdf.

[8] Ron Donagi and Roy Campbell Smith. The structure of the Prym map. Acta Math., 146(1-2):25-102, 1981.

[9] Carel Faber and Gerard van der Geer. Complete subvarieties of moduli spaces and the Prym map. $J$. Reine Angew. Math., 573:117-137, 2004.

[10] Gavril Farkas. Prym varieties and their moduli. In Contributions to algebraic geometry, EMS Ser. Congr. Rep., pages 215-255. Eur. Math. Soc., Zürich, 2012.

[11] Ernst Kani and Michael Rosen. Idempotent relations and factors of Jacobians. Math. Ann., 284(2):307$327,1989$.

[12] Toshiyuki Katsura and Frans Oort. Families of supersingular abelian surfaces. Compositio Math., 62(2):107-167, 1987.

[13] Nicholas M. Katz. Slope filtration of F-crystals. In Journées de Géométrie Algébrique de Rennes (Rennes, 1978), Vol. I, volume 63 of Astérisque, pages 113-163. Soc. Math. France, Paris, 1979.

[14] Herbert Lange and Angela Ortega. Prym varieties of cyclic coverings. Geom. Dedicata, 150:391-403, 2011.

[15] Shōichi Nakajima. On generalized Hasse-Witt invariants of an algebraic curve. In Galois groups and their representations (Nagoya, 1981), volume 2 of Adv. Stud. Pure Math., pages 69-88. North-Holland, Amsterdam, 1983.

[16] Florian Pop and Mohamed Saïdi. On the specialization homomorphism of fundamental groups of curves in positive characteristic. In Galois groups and fundamental groups, volume 41 of Math. Sci. Res. Inst. Publ., pages 107-118. Cambridge Univ. Press, Cambridge, 2003.

[17] Michel Raynaud. Sections des fibrés vectoriels sur une courbe. Bull. Soc. Math. France, 110(1):103-125, 1982.

[18] Michel Raynaud. Revêtements des courbes en caractéristique $p>0$ et ordinarité. Compositio Math., 123(1):73-88, 2000.

[19] Michel Raynaud. Sur le groupe fondamental d'une courbe complète en caractéristique $p>0$. In Arithmetic fundamental groups and noncommutative algebra (Berkeley, CA, 1999), volume 70 of Proc. Sympos. Pure Math., pages 335-351. Amer. Math. Soc., Providence, RI, 2002.

[20] Akio Tamagawa. On the tame fundamental groups of curves over algebraically closed fields of characteristic $>0$. In Galois groups and fundamental groups, volume 41 of Math. Sci. Res. Inst. Publ., pages 47-105. Cambridge Univ. Press, Cambridge, 2003.

[21] Alessandro Verra. The fibre of the Prym map in genus three. Math. Ann., 276(3):433-448, 1987.

[22] Angelo Vistoli. Intersection theory on algebraic stacks and on their moduli spaces. Invent. Math., 97(3):613-670, 1989.

[23] Noriko Yui. On the Jacobian varieties of hyperelliptic curves over fields of characteristic $p>2 . J$. Algebra, 52(2):378-410, 1978.

Bogazici University, Faculty of Arts and Sciences, Bebek, Istanbul, 34342, Turkey

E-mail address: ekin.ozman@boun.edu.tr

Department of Mathematics, Colorado State University, Fort Collins, CO 80523, USA

E-mail address: pries@math.colostate.edu 